\title{
Quadratic covariations for the solution to a stochastic heat equation with space-time white noise
}

\author{
Xichao Sun ${ }^{1} \mathbb{D}$, Litan Yan $^{2}$ and Xianye $\mathrm{Yu}^{3^{*}}$
}

\section{"Correspondence:}

xianyeyu@gmail.com

${ }^{3}$ School of Statistics and

Mathematics, Zhejiang Gongshang

University, Hangzhou, China

Full list of author information is

available at the end of the article

\section{Springer}

\begin{abstract}
Let $u(t, x)$ be the solution to a stochastic heat equation

$$
\frac{\partial}{\partial t} u=\frac{1}{2} \frac{\partial^{2}}{\partial x^{2}} u+\frac{\partial^{2}}{\partial t \partial x} X(t, x), \quad t \geq 0, x \in \mathbb{R}
$$

with initial condition $u(0, x) \equiv 0$, where $\dot{X}$ is a space-time white noise. This paper is an attempt to study stochastic analysis questions of the solution $u(t, x)$. In fact, it is well known that the solution is a Gaussian process such that the process $t \mapsto u(t, x)$ is a bi-fractional Brownian motion with Hurst indices $H=K=\frac{1}{2}$ for every real number $x$. However, the many properties of the process $x \mapsto u(\cdot, x)$ are unknown. In this paper we consider the generalized quadratic covariations of the two processes $x \mapsto u(\cdot, x), t \mapsto u(t, \cdot)$. We show that $x \mapsto u(\cdot, x)$ admits a nontrivial finite quadratic variation and the forward integral of some adapted processes with respect to it coincides with "Itô's integral", but it is not a semimartingale. Moreover, some generalized Itô formulas and Bouleau-Yor identities are introduced.
\end{abstract}

MSC: $60 \mathrm{G} 15 ; 60 \mathrm{H} 05 ; 60 \mathrm{H} 15$

Keywords: Fractional Brownian motion; Stochastic heat equation; Itô formula; Quadratic covariation; Local time

\section{Introduction}

Let $u(t, x)$ denote the solution to the stochastic heat equation

$$
\frac{\partial}{\partial t} u=\frac{1}{2} \frac{\partial^{2}}{\partial x^{2}} u+\frac{\partial^{2}}{\partial t \partial x} X(t, x), \quad t \geq 0, x \in \mathbb{R}^{d},
$$

with initial condition $u(0, x) \equiv 0$, where $\dot{X}$ is a space-time white noise on $[0, \infty) \times \mathbb{R}^{d}$, which are generalized Gaussian processes with covariance given by

$$
E[\dot{X}(t, x) \dot{X}(s, y)]=\delta(t-s) \delta(x-y) .
$$

(c) The Author(s) 2020. This article is licensed under a Creative Commons Attribution 4.0 International License, which permits use sharing, adaptation, distribution and reproduction in any medium or format, as long as you give appropriate credit to the original author(s) and the source, provide a link to the Creative Commons licence, and indicate if changes were made. The images or other third party material in this article are included in the article's Creative Commons licence, unless indicated otherwise in a credit line to the material. If material is not included in the article's Creative Commons licence and your intended use is not permitted by statutory regulation or exceeds the permitted use, you will need to obtain permission directly from the copyright holder. To view a copy of this licence, visit http://creativecommons.org/licenses/by/4.0/. 
Clearly, we have

$$
u(t, x)=\int_{0}^{t} \int_{\mathbb{R}^{d}} p(t-r, x-y) X(d r, d y)
$$

where $p(t, x)=\frac{1}{\sqrt{2 \pi t}} e^{-\frac{|x|^{2}}{2 t}}$ is the heat kernel. Then the processes $(t, x) \mapsto u(t, x), t \mapsto u(t, \cdot)$ and $x \mapsto u(\cdot, x)$ are Gaussian. Mueller and Tribe [19] were first to find that

$$
\begin{aligned}
E\left[[u(t, x)-u(t, y)]^{2}\right] & =\int_{0}^{t} \int_{\mathbb{R}}[p(r, x-z)-p(r, y-z)]^{2} d r d z \\
& \longrightarrow \int_{0}^{\infty} \int_{\mathbb{R}}[p(r, x-z)-p(r, y-z)]^{2} d r d z=C|x-y|
\end{aligned}
$$

for all $x, y \in \mathbb{R}^{d}$, as $t$ tends to infinity, if the initial value $u(0, x)=0$. By taking a special initial value (a two-sided $\mathbb{R}^{d}$-valued Brownian motion), the authors studied the stationary pinned string and hitting probabilities of a random string. When $d=1$ Swanson [30] has showed that (see also Pospisil and Tribe [25])

$$
E[u(t, x) u(s, x)]=\frac{1}{\sqrt{2 \pi}}\left((t+s)^{1 / 2}-|t-s|^{1 / 2}\right), \quad t, s \geq 0,
$$

and the process $t \mapsto u(t, x)$ has a nontrivial quartic variation. This shows that, for every $x \in \mathbb{R}$, the process $t \mapsto u(t, x)$ coincides with the bi-fractional Brownian motion and it is not a semimartingale, so a stochastic integral with respect to the process $t \mapsto u(t, x)$ cannot be defined in the classical Itô sense. Some surveys and complete literature for bifractional Brownian motion can be found in Houdré and Villa [13], Lei and Nualart [16], Russo and Tudor [27], Tudor and Xiao [33] and Yan et al. [35], and the references therein. Moreover, for more general parabolic SPDEs, many authors studied the regularity results and stochastic calculus with respect to their solutions. We mention the work of Balan and Kim [2], Da Prato et al. [4], Deya and Tindel [5], Gradinaru et al. [12], Lanconelli [14, 15], León and Tindel et al. [17], Nualart and Vuillermot [21], Ouahhabi and Tudor [22], Pardoux [23], Pospisil and Tribe [25], Torres et al. [31], Tudor [32], Tudor and Xiao [34], Zambotti [38], and the references therein.

In this paper, as an attempt we study stochastic analysis questions of the solution process $u=\{u(t, x), t \in[0, T], x \in \mathbb{R}\}$ of (1.1) with $u(0, x)=0$ associated with quadratic variation. We shall see (in Sect. 3) that the process $x \mapsto u(\cdot, x)$ admits a nontrivial finite quadratic variation in any finite interval, and, moreover, we shall also see (in Sect. 4) that the forward integral of some adapted processes with respect to $x \mapsto u(\cdot, x)$ coincides with "Itô's integral". On the other hand, as a noise, the stochastic process $u=\{u(t, x), t \geq 0, x \in \mathbb{R}\}$ is very rough in time and it is not white in space. However, the process $x \mapsto u(\cdot, x)$ admits some characteristics similar to Brownian motion. These results, together with the work of Mueller and Tribe [19], Pospisil and Tribe [25], and Swanson [30] point out that the process $u=\{u(t, x)\}$ as a noise admits the next special structures:

- It is very rough in time and similar to fractional Brownian motion with Hurst index $H=\frac{1}{4}$, but it has not stationary increments.

- It is not white in space, but its quadratic variation coincides with the classical Brownian motion and it is not self-similar. 
- The process in space variable is not a semimartingale, but the forward integral of some adapted processes with respect to the process in space variable coincides with "Itồs integral".

- The process $u=\{u(t, x)\}$ admits a simple representation via a Wiener integral with respect to the Brownian sheet.

- Though the process $u=\{u(t, x)\}$ is Gaussian, as a noise, its time and space parts are farraginous. We cannot decompose its covariance as the product of two independent parts. This is very different from fractional noise and white noise. In fact, we have

$$
E u(t, x) u(s, y)=\frac{1}{\sqrt{2 \pi}} \int_{0}^{s} \frac{1}{\sqrt{t+s-2 r}} \exp \left\{-\frac{(x-y)^{2}}{2(t+s-2 r)}\right\} d r
$$

for all $t \geq s>0$ and $x, y \in \mathbb{R}$.

Therefore, it seems interesting to study the integrals

$$
\int_{\mathbb{R}} f(x) u(t, d x), \quad \int_{0}^{t} f(s) u(d s, x), \quad \int_{0}^{t} \int_{\mathbb{R}} f(s, x) u(d s, d x),
$$

and some related stochastic (partial) differential equations. For example, one can consider the following "iterated" stochastic partial differential equations:

$$
\frac{\partial}{\partial t} u^{j}=\frac{1}{2} \frac{\partial^{2}}{\partial x^{2}} u^{j}+f\left(u^{j}\right)+\frac{\partial^{2}}{\partial t \partial x} u^{j-1}(t, x), \quad t \geq 0, x \in \mathbb{R}, j=1,2, \ldots,
$$

where $u^{0}$ is a space-time white noise. Of course, one can also consider some sample path properties and singular integrals

$$
\int_{I_{x}} d x \int_{0}^{t} \frac{1}{(u(s, x))_{+}^{1+\alpha}} d \sqrt{s}, \quad \alpha \geq 0
$$

with $t \geq 0$ and $I_{x}=[0, x]$ for $x \geq 0$ and $I_{x}=[x, 0]$ for $x \leq 0$. We will carry out these projects in some forthcoming work. In the present paper our objects are to study the quadratic covariations of $x \mapsto u(\cdot, x)$ and $t \mapsto u(t, \cdot)$, and introduce some generalized Itô formulas associated with $\{u(\cdot, x), x \in \mathbb{R}\}$ and $\{u(t, \cdot), t \geq 0\}$, respectively, and moreover we also consider their local times and Bouleau-Yor's identities.

To expound our aim, let us start with a basic definition. Let $u=\{u(t, x), t \in[0, T], x \in \mathbb{R}\}$ be the solution process of (1.1) with $u(0, x)=0$ and denote

$$
\Delta_{1}(s, t):=\sqrt{2 t}+\sqrt{2 s}+2 \sqrt{|t-s|}-2 \sqrt{t+s}
$$

and

$$
\Delta_{2}(s, t, z):=\int_{0}^{s \wedge t} \frac{2}{\sqrt{t+s-2 r}}\left(1-\exp \left\{-\frac{z^{2}}{2(t+s-2 r)}\right\}\right) d r
$$

for all $t, s>0$ and $z \geq 0$. An elementary calculation can show that

$$
E\left[(u(t, x)-u(s, y))^{2}\right]=\frac{1}{\sqrt{2 \pi}}\left[\Delta_{1}(s, t)+\Delta_{2}(s, t, x-y)\right]
$$


for all $t, s>0$ and $x, y \in \mathbb{R}$. This simple estimate inspires us to consider the following limits:

$$
\begin{aligned}
\lim _{\varepsilon \rightarrow 0} \frac{1}{\varepsilon} E\left[(u(t, x+\varepsilon)-u(t, x))^{2}\right] & =\frac{1}{\sqrt{\pi}} \lim _{\varepsilon \rightarrow 0} \frac{1}{\varepsilon} \int_{0}^{t} \frac{1}{\sqrt{r}}\left(1-e^{-\frac{\varepsilon^{2}}{4 r}}\right) d r \\
& =\frac{2}{\sqrt{\pi}} \int_{0}^{\infty} \frac{1}{s^{2}}\left(1-e^{-\frac{s^{2}}{4}}\right) d s=1
\end{aligned}
$$

and

$$
\lim _{\varepsilon \rightarrow 0} \frac{1}{\sqrt{\varepsilon}} E\left[(u(t+\varepsilon, x)-u(t, x))^{2}\right]=\sqrt{\frac{2}{\pi}}
$$

for all $t \geq 0$ and $x \in \mathbb{R}$. That is,

$$
\begin{aligned}
& \lim _{\delta \rightarrow 0} \lim _{\varepsilon \rightarrow 0} \frac{1}{\sqrt{\varepsilon}+\delta} E\left[(u(t+\varepsilon, x+\delta)-u(t, x))^{2}\right]=1, \\
& \lim _{\varepsilon \rightarrow 0} \lim _{\delta \rightarrow 0} \frac{1}{\sqrt{\varepsilon}+\delta} E\left[(u(t+\varepsilon, x+\delta)-u(t, x))^{2}\right]=\sqrt{\frac{2}{\pi}}
\end{aligned}
$$

for all $t \geq 0$ and $x \in \mathbb{R}$. Thus, the next definition is natural.

Definition 1.1 Denote $B:=\left\{B_{t}:=u(t, \cdot), t \geq 0\right\}$ and $W:=\left\{W_{x}:=u(\cdot, x), x \in \mathbb{R}\right\}$. Let $I_{x}=$ $[0, x]$ for $x \geq 0$ and $I_{x}=[x, 0]$ for $x \leq 0$. Define the integrals

$$
\begin{aligned}
& I_{\delta}^{1}(f, x, t)=\frac{1}{\delta} \int_{I_{x}}\left\{f\left(W_{y+\delta}\right)-f\left(W_{y}\right)\right\}\left(W_{y+\delta}-W_{y}\right) d y, \\
& I_{\varepsilon}^{2}(f, x, t)=\frac{1}{\sqrt{\varepsilon}} \int_{0}^{t}\left\{f\left(B_{s+\varepsilon}\right)-f\left(B_{s}\right)\right\}\left(B_{s+\varepsilon}-B_{s}\right) \frac{d s}{2 \sqrt{s}},
\end{aligned}
$$

for all $t \geq 0, x \in \mathbb{R}, \varepsilon, \delta>0$, where $f$ is a measurable function on $\mathbb{R}$. The $\operatorname{limits}_{\lim } \lim _{\delta \rightarrow 0} I_{\delta}^{1}(f$, $t, x)$ and $\lim _{\varepsilon \rightarrow 0} I_{\varepsilon}^{2}(f, t, x)$ are called the partial quadratic covariations (PQC, in short) in space and in time, respectively, of $f(u)$ and $u$, provided these limits exist in probability. We denote them by $[f(W), W]_{x}^{(S Q)}$ and $[f(B), B]_{t}^{(T Q)}$, respectively.

Clearly, we have (see Sect. 3)

$$
[f(W), W]_{x}^{(S Q)}=\int_{I_{x}} f^{\prime}\left(W_{y}\right) d y
$$

and $[W, W]_{x}^{(S Q)}=|x|$ for all $f \in C^{1}(\mathbb{R}), t>0, x \in \mathbb{R}$. We also have (see Sect. 6)

$$
[f(B), B]_{t}^{(T Q)}=\int_{0}^{t} f^{\prime}\left(B_{s}\right) \frac{d s}{\sqrt{2 \pi s}}
$$

and $[B, B]_{t}^{(T Q)}=\sqrt{\frac{2}{\pi} t}$ for all $f \in C^{1}(\mathbb{R}), t \geq 0, x \in \mathbb{R}$. These expressions say that the process $W=\left\{W_{x}=u(\cdot, x), x \in \mathbb{R}\right\}$ admits a nontrivial finite quadratic variation in any finite interval $I_{x}$. This is also a main motivation to study the solution of (1.1). More work for stochastic calculus with respect to a continuous finite quadratic variation process can be found in Errami and Russo [7] and Russo and Vallois [29]. 
This paper is organized as follows. In Sect. 2, we establish some technical estimates associated with the solution of (1.1), and as some applications we introduce Wiener integrals with respect to the two processes $B=\left\{B_{t}=u(t, \cdot), t \geq 0\right\}$ and $W=\left\{W_{x}=u(\cdot, x), x \in \mathbb{R}\right\}$, respectively. In Sect. 3 we show that the quadratic variation $[W, W]^{(S Q)}$ exists in $L^{2}(\Omega)$ and equals $|x|$ in every finite interval $I_{x}$. For a given $t>0$, by estimating in $L^{2}$

$$
\frac{1}{\varepsilon} \int_{I_{x}} f\left(W_{y+\varepsilon}\right)\left(W_{y+\varepsilon}-W_{y}\right) d y \quad(x \in \mathbb{R})
$$

and

$$
\frac{1}{\varepsilon} \int_{I_{x}} f\left(W_{y}\right)\left(W_{y+\varepsilon}-W_{y}\right) d y \quad(x \in \mathbb{R})
$$

for all $\varepsilon>0$, respectively, we construct a Banach space $\mathscr{H}_{t}$ of measurable functions such that the PQC $[f(W), W]^{(S Q)}$ in space exists in $L^{2}(\Omega)$ for all $f \in \mathscr{H}_{t}$, and in particular we have

$$
[f(W), W]_{x}^{(S Q)}=\int_{I_{x}} f^{\prime}\left(W_{y}\right) d y
$$

provided $f \in C^{1}(\mathbb{R})$. In Sect. 4, as an application of Sect. 3, we show that the Itô's formula

$$
F\left(W_{x}\right)=F\left(W_{0}\right)+\int_{I_{x}} f\left(W_{y}\right) \delta W_{y}+\frac{1}{2}[f(W), W]_{x}^{(S Q)}
$$

holds for all $t>0, x \in \mathbb{R}$, where the integral $\int_{I_{x}} f^{\prime}\left(W_{y}\right) \delta W_{y}$ denotes the Skorohod integral, $F$ is an absolutely continuous function with the derivative $F^{\prime}=f \in \mathscr{H}_{t}$. In order to obtain the above Itô formula, we first introduce a standard Itô type formula

$$
F\left(W_{x}\right)=F\left(W_{0}\right)+\int_{I_{x}} F^{\prime}\left(W_{y}\right) \delta W_{y}+\frac{1}{2} \int_{I_{x}} F^{\prime \prime}\left(W_{y}\right) d y
$$

for all $F \in C^{2}(\mathbb{R})$ satisfying some suitable conditions. It is important to note that the Gaussian process $W=\left\{W_{x}=u(\cdot, x), x \in \mathbb{R}\right\}$ does not satisfy the condition in Alós et al. [1] since

$$
E\left[u(t, x)^{2}\right]=\sqrt{\frac{t}{\pi}}, \frac{d}{d x} E\left[u(t, x)^{2}\right]=0
$$

for all $t \geq 0$ and $x \in \mathbb{R}$. We need to give the proof of Eq. (1.6). Moreover, we also show that the forward integral (see Russo-Vallois [29])

$$
\int_{I_{x}} f\left(W_{y}\right) d^{-} W_{y}:=\operatorname{ucp} \lim _{\varepsilon \downarrow 0} \frac{1}{\varepsilon} \int_{I_{x}} f\left(W_{y}\right)\left(W_{y+\varepsilon}-W_{y}\right) d y
$$

coincides with the Skorohod integral $\int_{I_{x}} f\left(W_{y}\right) \delta W_{y}$, if $f$ satisfies the growth condition

$$
|f(y)| \leq C e^{\beta y^{2}}, \quad y \in \mathbb{R}
$$

with $0 \leq \beta<\frac{\sqrt{\pi}}{4 \sqrt{t}}$, where the notation ucp lim denotes the uniform convergence in probability on each compact interval. This is very similar to Brownian motion, but the process 
$W=\left\{W_{x}=u(\cdot, x), x \in \mathbb{R}\right\}$ is not a semimartingale. In Sect. 5 we consider some questions associated with the local time

$$
\mathscr{L}^{t}(x, a)=\int_{0}^{x} \delta\left(W_{y}-a\right) d y
$$

of the process $W=\left\{W_{x}=u(\cdot, x), x \geq 0\right\}$. In particular, we show that the Bouleau-Yor type identity

$$
[f(W), W]_{x}^{(S Q)}=-\int_{\mathbb{R}} f(v) \mathscr{L}^{t}(x, d v)
$$

holds for all $f \in \mathscr{H}_{t}$. In Sect. 6 we consider some analysis questions associated with the quadratic covariation of the process $B=\left\{B_{t}=u(t, \cdot), t \geq 0\right\}$.

\section{Some basic estimates}

In this section we will establish divergence integral and some technical estimates associated with the solution

$$
u(t, x)=\int_{0}^{t} \int_{\mathbb{R}} p(t-r, x-y) X(d r, d y), \quad t \geq 0, x \in \mathbb{R},
$$

where $p(t, x)=\frac{1}{\sqrt{2 \pi t}} e^{-\frac{x^{2}}{2 t}}$ is the heat kernel. Recall that the temporal process $B=\left\{B_{t}=\right.$ $u(t, \cdot), t \geq 0\}$ and spatial process $W=\left\{W_{x}=u(\cdot, x), x \in \mathbb{R}\right\}$. As mentioned earlier, the temporal process $B=\left\{B_{t}\right\}$ is neither a semimartingale nor a Markov process. Moreover, from the next discussion we shall also see that the spatial process $W=\left\{W_{x}\right\}$ is neither a semimartingale nor a Markov process. However, as two Gaussian processes, one can develop the stochastic calculus of variations with respect to them. We refer to Alós et al. [1], Nualart [20] and the references therein for more details of stochastic calculus of Gaussian process.

As usual, we assume that $\mathcal{H}_{\Delta}$ and $\mathcal{H}_{\nabla}$ are the reproducing kernel Hilbert spaces associated with $W=\left\{W_{x}=u(\cdot, x), x \in[-M, M]\right\}$ and $B=\left\{B_{t}=u(t, \cdot), t \in[0, T]\right\}$, respectively. Then the Wiener integrals

$$
W(\varphi):=\int_{-M}^{M} \varphi(x) d W_{x}, \quad \varphi \in \mathcal{H}_{\Delta}
$$

and

$$
B(\varphi):=\int_{0}^{T} \psi(s) d B_{s}, \quad \psi \in \mathcal{H}_{\nabla}
$$

exist as two Gaussian random variables, and

$$
E\left(\int_{-M}^{M} \varphi_{1}(x) d W_{x} \int_{-M}^{M} \varphi_{2}(x) d W_{x}\right)=\left\langle\varphi_{1}, \varphi_{2}\right\rangle_{\mathcal{H}_{\Delta}}
$$

for $\varphi_{1}, \varphi_{2} \in \mathcal{H}_{\triangle}$ and

$$
E\left(\int_{0}^{T} \psi_{1}(s) d B_{s} \int_{0}^{T} \psi_{2}(s) d B_{s}\right)=\left\langle\psi_{1}, \psi_{2}\right\rangle_{\mathcal{H}_{\nabla}}
$$


for $\psi_{1}, \psi_{2} \in \mathcal{H}_{\nabla}$. Clearly, for $t, s>0$ and $x, y \in \mathbb{R}$ we have

$$
\left\langle 1_{I_{x}}, 1_{I_{y}}\right\rangle_{\mathcal{H}_{\Delta}}=E\left(W_{x} W_{y}\right)=\frac{1}{2 \sqrt{\pi}} \int_{0}^{t} \frac{1}{\sqrt{r}} \exp \left\{-\frac{(x-y)^{2}}{4 r}\right\} d r
$$

and

$$
\left\langle 1_{[0, t]}, 1_{[0, s]}\right\rangle_{\mathcal{H}_{\nabla}}=E\left(B_{t} B_{s}\right)=\frac{1}{\sqrt{2 \pi}}\left((t+s)^{1 / 2}-|t-s|^{1 / 2}\right) .
$$

Denote by $\mathcal{S}_{\triangle}$ and $\mathcal{S}_{\nabla}$ the sets of smooth functionals of the form

$$
F_{\Delta}=f\left(W\left(\varphi_{1}\right), W\left(\varphi_{2}\right), \ldots, W\left(\varphi_{n}\right)\right)
$$

and

$$
F_{\nabla}=f\left(B\left(\psi_{1}\right), B\left(\psi_{2}\right), \ldots, B\left(\psi_{n}\right)\right),
$$

where $f \in C_{b}^{\infty}\left(\mathbb{R}^{n}\right)(f$ and all their derivatives are bounded $), \varphi_{i} \in \mathcal{H}_{\Delta}$ and $\psi_{i} \in \mathcal{H}_{\nabla}$. The derivative operators $D^{\Delta}$ and $D^{\nabla}$ (the Malliavin derivatives) of functionals $F_{t}$ and $F_{*}$ of the above forms are defined as

$$
D^{\Delta} F_{\Delta}=\sum_{j=1}^{n} \frac{\partial f}{\partial x_{j}}\left(W\left(\varphi_{1}\right), W\left(\varphi_{2}\right), \ldots, W\left(\varphi_{n}\right)\right) \varphi_{j}
$$

and

$$
D^{\nabla} F_{\nabla}=\sum_{j=1}^{n} \frac{\partial f}{\partial x_{j}}\left(B\left(\psi_{1}\right), B\left(\psi_{2}\right), \ldots, B\left(\psi_{n}\right)\right) \psi_{j},
$$

respectively. These derivative operators $D^{\Delta}, D^{\nabla}$ are then closable from $L^{2}(\Omega)$ into $L^{2}(\Omega$; $\left.\mathcal{H}_{\triangle}\right)$ and $L^{2}\left(\Omega ; \mathcal{H}_{*}\right)$, respectively. We denote by $\mathbb{D}^{\Delta, 1,2}$ and $\mathbb{D}^{\nabla, 1,2}$ the closures of $\mathcal{S}_{\triangle}$ and $\mathcal{S}_{\nabla}$ with respect to the norms

$$
\left\|F_{\Delta}\right\|_{\triangle, 1,2}:=\sqrt{E|F|^{2}+E\left\|D^{\Delta} F_{\Delta}\right\|_{\mathcal{H}_{\Delta}}^{2}}
$$

and

$$
\left\|F_{\nabla}\right\|_{\nabla, 1,2}:=\sqrt{E|F|^{2}+E\left\|D^{\nabla} F_{\nabla}\right\|_{\mathcal{H}_{\nabla}}^{2}},
$$

respectively. The divergence integrals $\delta^{\Delta}$ and $\delta^{\nabla}$ are the adjoint of derivative operators $D^{\Delta}$ and $D^{\nabla}$, respectively. We say that random variables $v \in L^{2}\left(\Omega ; \mathcal{H}_{\Delta}\right)$ and $w \in L^{2}\left(\Omega ; \mathcal{H}_{\nabla}\right)$ belong to the domains of the divergence operators $\delta^{\Delta}$ and $\delta^{\nabla}$, respectively, denoted by $\operatorname{Dom}\left(\delta^{\triangle}\right)$ and $\operatorname{Dom}\left(\delta^{\nabla}\right)$ if

$$
E\left|\left\langle D^{\Delta} F_{\Delta}, v\right\rangle_{\mathcal{H}_{\Delta}}\right| \leq c\left\|F_{\Delta}\right\|_{L^{2}(\Omega)}, E\left|\left\langle D^{\nabla} F_{\nabla}, w\right\rangle_{\mathcal{H}_{*}}\right| \leq c\left\|F_{\nabla}\right\|_{L^{2}(\Omega)},
$$


respectively, for all $F_{\Delta} \in \mathcal{S}_{\Delta}$ and $F_{\nabla} \in \mathcal{S}_{\nabla}$. In these cases $\delta^{\Delta}(v)$ and $\delta^{\nabla}(w)$ are defined by the duality relationships

$$
\begin{aligned}
E\left[F_{\Delta} \delta^{\Delta}(v)\right] & =E\left\langle D^{\Delta} F_{\Delta}, v\right\rangle_{\mathcal{H}_{\Delta}}, \\
E\left[F_{\nabla} \delta^{\nabla}(w)\right] & =E\left\langle D^{\nabla} F_{\nabla}, w\right\rangle_{\mathcal{H}_{\nabla}},
\end{aligned}
$$

respectively, for any $v \in \mathbb{D}^{\Delta, 1,2}$ and $w \in \mathbb{D}^{\nabla, 1,2}$. We have $\mathbb{D}^{\Delta, 1,2} \subset \operatorname{Dom}\left(\delta^{\Delta}\right)$ and $\mathbb{D}^{\nabla, 1,2} \subset$ $\operatorname{Dom}\left(\delta^{\nabla}\right)$. We will use the notations

$$
\delta^{\Delta}(v)=\int_{\mathbb{R}} v_{y} \delta W_{y}, \delta^{\nabla}(w)=\int_{0}^{T} w_{s} \delta B_{s}
$$

to express the Skorohod integrals, and the indefinite Skorohod integrals are defined as

$$
\int_{I_{x}} v_{y} \delta W_{y}=\delta^{\Delta}\left(v 1_{I_{x}}\right), \int_{0}^{t} w_{s} \delta B_{s}=\delta^{\nabla}\left(w 1_{[0, t]}\right)
$$

respectively. It is important to note that we can localize the domains of the operators $D^{\Delta}, D^{\nabla}, \delta^{\Delta}$ and $\delta^{\nabla}$ via the usual manner. If $\mathbb{L}$ is a class of random variables (or processes) we denote by $\mathbb{L}_{\text {loc }}$ the set of random variables $F$ such that there exists a sequence $\left\{\left(\Omega_{n}, F^{n}\right), n \geq 1\right\} \subset \mathscr{F} \times \mathbb{L}$ with the following properties:

(i) $\Omega_{n} \uparrow \Omega$,

(ii) $F=F^{n}$ a.s. on $\Omega_{n}$.

Take the operators $D^{\Delta}$ and $\delta^{\Delta}$ for instance. If $F \in \mathbb{D}_{\text {loc }}^{\Delta, 1,2}$, and $\left(\Omega_{n}, F^{n}\right)$ localizes $F$ in $\mathbb{D}^{\Delta, 1,2}$, then $D^{\Delta} F$ is defined without ambiguity by $D^{\Delta} F=D^{\Delta} F^{n}$ on $\Omega_{n}, n \geq 1$. Then, if $v \in \mathbb{D}_{\text {loc }}^{\Delta, 1,2}$, the divergence $\delta^{\Delta}(v)$ is defined as a random variable determined by the conditions

$$
\left.\delta^{\Delta}(v)\right|_{\Omega_{n}}=\left.\delta^{\Delta}\left(v^{n}\right)\right|_{\Omega_{n}} \quad \text { for all } n \geq 1,
$$

where $\left(\Omega_{n}, v^{n}\right)$ is a localizing sequence for $v$, but it may depend on the localizing sequence.

At the end of this section, we introduce some estimates for the spatial process $W=\left\{W_{x}=\right.$ $u(\cdot, x)\}$ and temporal processes $B=\left\{B_{t}=u(t, \cdot)\right\}$. For simplicity throughout this paper we let $C$ stand for a positive constant depending only on the subscripts and its value may be different at different places, and this assumption also holds true for $c$. Moreover, the notation $F \asymp G$ means that there are positive constants $c_{1}$ and $c_{2}$ such that

$$
c_{1} G(x) \leq F(x) \leq c_{2} G(x)
$$

in the common domain of definition for $F$ and $G$. We have

$$
\begin{aligned}
R_{x, y}(s, t): & =E u(t, x) u(s, y)=\int_{0}^{s} \int_{\mathbb{R}} p(t-r, x-z) p(s-r, y-z) d z d r \\
& =\frac{1}{2 \pi} \int_{0}^{s} \int_{\mathbb{R}} \frac{1}{\sqrt{(t-r)(s-r)}} \exp \left\{-\frac{(x-z)^{2}}{2(t-r)}-\frac{(y-z)^{2}}{2(s-r)}\right\} d z d r \\
& =\frac{1}{\sqrt{2 \pi}} \int_{0}^{s} \frac{1}{\sqrt{t+s-2 r}} \exp \left\{-\frac{(x-y)^{2}}{2(t+s-2 r)}\right\} d r
\end{aligned}
$$


for all $t \geq s>0, x, y \in \mathbb{R}$ and

$$
E\left[(u(t, x)-u(s, y))^{2}\right]=\frac{1}{\sqrt{2 \pi}}\left[\Delta_{1}(t, s)+\Delta_{2}(s, t, x-y)\right)
$$

for all $t>s>0$ and $x, y \in \mathbb{R}$, where

$$
\Delta_{1}(s, t):=\sqrt{2 t}+\sqrt{2 s}+2 \sqrt{|t-s|}-2 \sqrt{t+s}
$$

and

$$
\Delta_{2}(s, t, z):=\int_{0}^{s \wedge t} \frac{2}{\sqrt{t+s-2 r}}\left(1-\exp \left\{-\frac{z^{2}}{2(t+s-2 r)}\right\}\right) d r
$$

for all $t, s>0$ and $z \geq 0$.

Lemma 2.1 For all $t \geq s>0$ and $x, y \in \mathbb{R}$ we have

$$
E\left[(u(t, x)-u(s, y))^{2}\right] \leq C(\sqrt{t-s}+|x-y|) .
$$

By taking a special initial value (a two-sided $\mathbb{R}^{d}$-valued Brownian motion), Mueller and Tribe [19] showed that the above estimate is reversible. However, when the initial value $u(0, x)=0$, the above estimate is not reversible unless $x=y$. In fact, we have

$$
\lim _{x \uparrow 1} \frac{\sqrt{2}+\sqrt{2 x}+2 \sqrt{1-x}-2 \sqrt{1+x}}{\sqrt{1-x}}=1,
$$

which implies that

$$
\begin{aligned}
0 \leq \Delta_{1}(t, s) & =(\sqrt{2 t}+\sqrt{2 s}+2 \sqrt{t-s}-2 \sqrt{t+s}) \\
& =\sqrt{t}(\sqrt{2}+\sqrt{2 u}+2 \sqrt{1-u}-2 \sqrt{1+u}) \asymp \sqrt{t} \sqrt{1-u}=\sqrt{t-s}
\end{aligned}
$$

with $u=\frac{s}{t}$ by the continuity. On the other hand, we also have

$$
\begin{aligned}
\Delta_{2}(s, t, x-y) & =\int_{0}^{s} \frac{2}{\sqrt{t+s-2 r}}\left(1-\exp \left\{-\frac{(x-y)^{2}}{2(t+s-2 r)}\right\}\right) d r \\
& =\mid x-y \int_{\frac{|x-y|}{\sqrt{t+s}}}^{\frac{|x-y|}{\sqrt{t-s}}}\left(1-e^{-\frac{r^{2}}{2}}\right) \frac{d r}{r^{2}} \\
& \leq|x-y| \int_{0}^{+\infty}\left(1-e^{-\frac{r^{2}}{2}}\right) \frac{d r}{r^{2}}=|x-y| \sqrt{\frac{\pi}{2}}
\end{aligned}
$$

for all $t \geq s>0$ and $u \in \mathbb{R}$. However, by the facts that

$$
1-e^{-x} \asymp \frac{x}{1+x}, \frac{\pi}{2}-\arctan x \asymp \frac{1}{1+x},
$$

for all $x \geq 0$, we have

$$
\Delta_{2}(t, t, z)=z \int_{\frac{z}{\sqrt{2 t}}}^{\infty}\left(1-e^{-\frac{r^{2}}{2}}\right) \frac{d r}{r^{2}}
$$




$$
\asymp z\left(\frac{\pi}{2}-\arctan \frac{z}{\sqrt{2 t}}\right) \asymp \frac{z \sqrt{t}}{\sqrt{t}+z}
$$

for all $t>0$ and $z \geq 0$, and

$$
\Delta_{2}(s, t, z)=z \int_{\frac{z}{\sqrt{t+s}}}^{\frac{z}{t-s}}\left(1-e^{-\frac{r^{2}}{2}}\right) \frac{d r}{r^{2}} \asymp z\left(\arctan \frac{z}{\sqrt{t-s}}-\arctan \frac{z}{\sqrt{t+s}}\right)
$$

for $t>s \geq 0$ and $z \geq 0$.

Corollary 2.1 For all $t>0$ and $x, y \in \mathbb{R}$ we have

$$
E\left[(u(t, x)-u(t, y))^{2}\right] \asymp \frac{|x-y| \sqrt{t}}{\sqrt{t}+|x-y|} .
$$

Lemma 2.2 For all $t, s, r>0$ and $x \in \mathbb{R}$ we have

$$
|E[u(r, x)(u(t, x)-u(s, x))]| \leq 3 \sqrt{|t-s|} .
$$

Proof For all $t, s, r>0$ and $x \in \mathbb{R}$, we have

$$
\begin{aligned}
|E[u(r, x)(u(t, x)-u(s, x))]| & =|E u(r, x) u(t, x)-E u(r, x) u(s, x)| \\
& =\left|R_{x, x}(r, t)-R_{x, x}(r, s)\right| \\
& =\frac{1}{\sqrt{2 \pi}}|\sqrt{t+r}-\sqrt{|t-r|}-\sqrt{s+r}+\sqrt{|s-r|}| \\
& \leq|\sqrt{t+r}-\sqrt{s+r}|+|\sqrt{|t-r|}-\sqrt{|s-r|}| \leq 3 \sqrt{|t-s|}
\end{aligned}
$$

for all $t, s, r>0$ and $x \in \mathbb{R}$.

Lemma 2.3 For all $t>0$ and $x, y, z \in \mathbb{R}$ we have

$$
|E[u(t, x)(u(t, y)-u(t, z))]| \leq C|y-z|
$$

Proof For all $t>0$ and $x, y, z \in \mathbb{R}$, we have

$$
\begin{aligned}
E[ & u(t, x)(u(t, y)-u(t, z))] \\
& =E u(t, x) u(t, y)-E u(t, x) u(t, z) \\
& =R_{x, y}(t, t)-R_{x, z}(t, t) \\
& =\frac{1}{2 \sqrt{\pi}}\left(\int_{0}^{t} \frac{1}{\sqrt{t-r}} \exp \left\{-\frac{(x-y)^{2}}{4(t-r)}\right\} d r-\int_{0}^{t} \frac{1}{\sqrt{t-r}} \exp \left\{-\frac{(x-z)^{2}}{4(t-r)}\right\} d r\right) \\
& =\frac{\sqrt{t}}{2 \sqrt{\pi}}\left(|x-y| \int_{|x-y|}^{+\infty} \frac{1}{s^{2}} e^{-\frac{s^{2}}{4 t}} d s-|x-z| \int_{|x-z|}^{+\infty} \frac{1}{s^{2}} e^{-\frac{s^{2}}{4 t}} d s\right) .
\end{aligned}
$$

Consider the function $f: \mathbb{R}_{+} \rightarrow \mathbb{R}_{+}$defined by

$$
f(x)=x \int_{x}^{+\infty} \frac{1}{s^{2}} e^{-\frac{s^{2}}{4 t}} d s=e^{-\frac{x^{2}}{4 t}}-\frac{x}{2 t} \int_{x}^{\infty} e^{-\frac{r^{2}}{4 t}} d r .
$$


Then, by the mean value theorem, we have

$$
\begin{aligned}
|f(u)-f(v)| & =\frac{1}{2 t}|u-v| \int_{\xi}^{+\infty} e^{-\frac{s^{2}}{4 t}} d s \\
& \leq \frac{1}{2 t}|u-v| \int_{0}^{+\infty} e^{-\frac{s^{2}}{4 t}} d s \leq \frac{\sqrt{\pi}}{2 \sqrt{t}}|u-v|
\end{aligned}
$$

for all $u, v \geq 0$ and some $\xi$ between $u$ and $v$. It follows that

$$
|E[u(t, x)(u(t, y)-u(t, z))]| \leq \frac{1}{4}|| x-y|-| x-z|| \leq \frac{1}{4}|y-z|
$$

for all $t>0$ and $x, y, z \in \mathbb{R}$.

Lemma 2.4 For all $t>s>t^{\prime}>s^{\prime}>0$ and $x \in \mathbb{R}$ we have

$$
\left|E\left[(u(t, x)-u(s, x))\left(u\left(t^{\prime}, x\right)-u\left(s^{\prime}, x\right)\right)\right]\right| \leq \frac{C\left(t^{\prime}-s^{\prime}\right) \sqrt{t-s}}{\sqrt{t s\left(s-s^{\prime}\right)\left(t-t^{\prime}\right)}}
$$

Proof For all $t>s>t^{\prime}>s^{\prime}>0$ and $x \in \mathbb{R}$ we have

$$
\begin{aligned}
E[ & \left.(u(t, x)-u(s, x))\left(u\left(t^{\prime}, x\right)-u\left(s^{\prime}, x\right)\right)\right] \\
= & R_{x, x}\left(t, t^{\prime}\right)-R_{x, x}\left(s, t^{\prime}\right)-R_{x, x}\left(t, s^{\prime}\right)+R_{x, x}\left(s, s^{\prime}\right) \\
= & \frac{1}{\sqrt{2 \pi}}\left(\sqrt{t+t^{\prime}}-\sqrt{t-t^{\prime}}-\sqrt{s+t^{\prime}}+\sqrt{s-t^{\prime}}\right. \\
& \left.-\sqrt{t+s^{\prime}}+\sqrt{t-s^{\prime}}+\sqrt{s+s^{\prime}}-\sqrt{s-s^{\prime}}\right) .
\end{aligned}
$$

Consider the function

$$
f(x)=\sqrt{t+x}-\sqrt{t-x}-\sqrt{s+x}+\sqrt{s-x}
$$

with $x \in[0, s]$. Then, by the mean value theorem, we have

$$
\begin{aligned}
\mid E & {\left[(u(t, x)-u(s, x))\left(u\left(t^{\prime}, x\right)-u\left(s^{\prime}, x\right)\right)\right] \mid } \\
& =\frac{1}{\sqrt{2 \pi}}\left|f\left(t^{\prime}\right)-f\left(s^{\prime}\right)\right| \\
& =\frac{1}{2}\left(t^{\prime}-s^{\prime}\right)\left|\frac{1}{\sqrt{t+\xi}}-\frac{1}{\sqrt{t-\xi}}-\frac{1}{\sqrt{s+\xi}}+\frac{1}{\sqrt{s-\xi}}\right| \\
& \leq \frac{1}{2}\left(t^{\prime}-s^{\prime}\right)\left(\frac{\sqrt{t+\xi}-\sqrt{s+\xi}}{\sqrt{t+\xi} \sqrt{s+\xi}}+\frac{\sqrt{t-\xi}-\sqrt{s-\xi}}{\sqrt{s-\xi} \sqrt{t-\xi}}\right) \\
& \leq \frac{C\left(t^{\prime}-s^{\prime}\right) \sqrt{t-s}}{\sqrt{t s\left(s-s^{\prime}\right)\left(t-t^{\prime}\right)}}
\end{aligned}
$$

for some $s^{\prime} \leq \xi \leq t^{\prime}$ and the lemma follows.

Lemma 2.5 For all $t>0$ and $x>y>x^{\prime}>y^{\prime}$ we have

$$
\left|E\left[(u(t, x)-u(t, y))\left(u\left(t, x^{\prime}\right)-u\left(t, y^{\prime}\right)\right)\right]\right| \leq \frac{1}{4 \sqrt{t \pi}}(x-y)\left(x^{\prime}-y^{\prime}\right) e^{-\frac{\left(y-x^{\prime}\right)^{2}}{4 t}} .
$$


Proof Define the function $f: \mathbb{R}_{+} \rightarrow \mathbb{R}_{+}$by

$$
f(x)=x \int_{x}^{+\infty} \frac{1}{s^{2}} e^{-\frac{s^{2}}{4 t}} d s .
$$

Then, similar to the proof of Lemma 2.3, we have

$$
\begin{aligned}
& E\left[(u(t, x)-u(t, y))\left(u\left(t, x^{\prime}\right)-u\left(t, y^{\prime}\right)\right)\right] \\
& \quad=R_{x, x^{\prime}}(t, t)-R_{x, y^{\prime}}(t, t)-R_{y, x^{\prime}}(t, t)+R_{y, y^{\prime}}(t, t) \\
& \quad=\frac{\sqrt{t}}{2 \sqrt{\pi}}\left[f\left(x-x^{\prime}\right)-f\left(x-y^{\prime}\right)-f\left(y-x^{\prime}\right)+f\left(y-y^{\prime}\right)\right]
\end{aligned}
$$

for all $t>0$ and $x>y>x^{\prime}>y^{\prime}$. By mean value theorem it follows that

$$
\begin{aligned}
\mid E & {\left[(u(t, x)-u(t, y))\left(u\left(t, x^{\prime}\right)-u\left(t, y^{\prime}\right)\right)\right] \mid } \\
& =\frac{\sqrt{t}}{2 \sqrt{\pi}}(x-y)\left|f^{\prime}\left(\xi-x^{\prime}\right)-f^{\prime}\left(\xi-y^{\prime}\right)\right| \\
& =\frac{1}{4 \sqrt{t} \sqrt{\pi}}(x-y)\left|\int_{\xi-x^{\prime}}^{+\infty} e^{-\frac{s^{2}}{4 t}} d s-\int_{\xi-y^{\prime}}^{+\infty} e^{-\frac{s^{2}}{4 t}} d s\right| \\
& =\frac{1}{2 \sqrt{\pi}}(x-y) \int_{\xi-x^{\prime}}^{\xi-y^{\prime}} \frac{1}{2 \sqrt{t}} e^{-\frac{s^{2}}{4 t}} d s \leq \frac{1}{4 \sqrt{t \pi}}(x-y)\left(x^{\prime}-y^{\prime}\right)
\end{aligned}
$$

for some $\xi \in[y, x]$. This completes the proof.

Lemma 2.6 For all $t>s>0$ and $x \in \mathbb{R}$ denote $\sigma_{t, x}^{2}=E\left[u(t, x)^{2}\right], \sigma_{s, x}^{2}=E\left[u(s, x)^{2}\right], \mu_{t, s, x}=$ $E[u(t, x) u(s, x)]$. Then we have

$$
\frac{1}{\pi} \sqrt{s(t-s)} \leq \sigma_{t, x}^{2} \sigma_{s, x}^{2}-\mu_{t, s, x}^{2} \leq \frac{3}{\pi} \sqrt{s(t-s)} .
$$

Proof Given $t>s>0$ and $x \in \mathbb{R}$, we have

$$
\begin{aligned}
\sigma_{t, x}^{2} \sigma_{s, x}^{2}-\mu_{t, s, x}^{2} & =\frac{1}{2 \pi}\left(2 \sqrt{t s}-\left((t+s)^{1 / 2}-(t-s)^{1 / 2}\right)^{2}\right) \\
& =\frac{1}{\pi}\left(\sqrt{t s}-t+\sqrt{t^{2}-s^{2}}\right)=\frac{t}{\pi}\left(\sqrt{z}+\sqrt{1-z^{2}}-1\right)
\end{aligned}
$$

with $z=\frac{s}{t}$. Elementary calculus can show that

$$
\sqrt{z}+\sqrt{1-z^{2}}-1 \asymp \sqrt{z(1-z)}
$$

for all $0 \leq z \leq 1$. In fact, we have

$$
\sqrt{z}+\sqrt{1-z^{2}}-1=\sqrt{z(1-z)}+\left(\sqrt{z}+\sqrt{1-z^{2}}-1-\sqrt{z(1-z)}\right) \geq \sqrt{z(1-z)}
$$

since $\sqrt{z}+\sqrt{1-z^{2}}-1-\sqrt{z(1-z)} \geq 0$. Conversely, we have also

$$
0 \leq \sqrt{z}+\sqrt{1-z}-1=\sqrt{z(1-z)}+\sqrt{z}+(\sqrt{1-z}-\sqrt{z(1-z)})-1
$$




$$
=\sqrt{z(1-z)}-(1-\sqrt{z})(1-\sqrt{1-z}) \leq \sqrt{z(1-z)},
$$

which gives

$$
\begin{aligned}
\sqrt{z}+\sqrt{1-z^{2}}-1 & =\sqrt{z}+\sqrt{1-z+z-z^{2}}-1 \\
& \leq \sqrt{z}+\sqrt{1-z}-1+\sqrt{z(1-z)} \leq 2 \sqrt{z(1-z)} .
\end{aligned}
$$

This completes the proof.

Lemma 2.7 For all $t>0$ and $x>y$ denote $\mu_{t, x, y}=E[u(t, x) u(t, y)]$. Then, under the conditions of Lemma 2.6, we have

$$
\sigma_{t, x}^{2} \sigma_{t, y}^{2}-\mu_{t, x, y}^{2} \asymp \frac{(x-y) t}{\sqrt{t}+x-y} .
$$

In particular, we have

$$
0 \leq \sigma_{t, z}^{2}-\mu_{t, x, y} \asymp \frac{|x-y| t}{\sqrt{t}+|x-y|}
$$

for all $t>0$ and $x, y, z \in \mathbb{R}$.

Proof Given $t>0$ and $x>y$. We have

$$
\begin{aligned}
\sigma_{t, x}^{2} \sigma_{t, y}^{2}-\mu_{t, x, y}^{2}= & \frac{t}{\pi}-\frac{1}{4 \pi}\left(\int_{0}^{t} \frac{1}{\sqrt{r}} \exp \left\{-\frac{(x-y)^{2}}{4 r}\right\} d r\right)^{2} \\
= & \frac{1}{4 \pi}\left(4 t-\left(\int_{0}^{t} \frac{1}{\sqrt{r}} \exp \left\{-\frac{(x-y)^{2}}{4 r}\right\} d r\right)^{2}\right) \\
= & \frac{1}{4 \pi} \int_{0}^{t} \frac{d r}{\sqrt{r}}\left(1-\exp \left\{-\frac{(x-y)^{2}}{4 r}\right\}\right) \\
& \times \int_{0}^{t} \frac{d r}{\sqrt{r}}\left(1+\exp \left\{-\frac{(x-y)^{2}}{4 r}\right\}\right) \\
\asymp & \sqrt{t} \int_{0}^{t} \frac{d r}{\sqrt{r}}\left(1-\exp \left\{-\frac{(x-y)^{2}}{4 r}\right\}\right) \\
= & \sqrt{t}(x-y) \int_{\frac{x-y}{\sqrt{t}}}^{\infty}\left(1-e^{-\frac{s^{2}}{4}}\right) \frac{d s}{s^{2}} \asymp \frac{(x-y) t}{\sqrt{t}+x-y}
\end{aligned}
$$

by (2.5) and the lemma follows.

3 The quadratic covariation of the spatial process $W=\left\{W_{x},|x| \leq M\right\}$

In this section, we study the existence of the PQC $[f(W), W]^{(S Q)}$. We fix a time parameter $t>0$. Recall that

$$
I_{\varepsilon}^{2}(f, x, t)=\frac{1}{\varepsilon} \int_{I_{x}}\left\{f\left(W_{y+\varepsilon}\right)-f\left(W_{y}\right)\right\}\left(W_{y+\varepsilon}-W_{y}\right) d y
$$


for $\varepsilon>0$ and $x \in \mathbb{R}$, and

$$
[f(W), W]_{x}^{(S Q)}=\lim _{\varepsilon \downarrow} I_{\varepsilon}^{2}(f, x, t),
$$

provided the limit exists in probability. In [25], Pospisil and Tribe have showed that the following convergence holds.

Lemma 3.1 Let $x \in \mathbb{R}$ and let $x_{j}^{n}=\frac{j x}{n} ; j=0,1, \ldots, n$. Then we have

$$
\sum_{j=1}^{n}\left(W_{x_{j}^{n}}-W_{x_{j-1}^{n}}\right)^{2} \longrightarrow|x|,
$$

in $L^{2}(\Omega)$, as $n$ tends to infinity.

In this section we are not only to show that

$$
[W, W]_{x}^{(S Q)}=\lim _{\varepsilon \downarrow 0} \frac{1}{\varepsilon} \int_{I_{x}}\left(W_{y+\varepsilon}-W_{y}\right)^{2} d y=|x|
$$

in $L^{2}$, but also to find a Banach space of Borel functions such that

$$
[f(W), W]_{x}^{(S Q)}
$$

exists in $L^{2}$ for all Borel functions $f$ belonging to the Banach space.

Proposition 3.1 We have

$$
[f(W), W]_{x}^{(S Q)}=\int_{I_{x}} f^{\prime}\left(W_{y}\right) d y
$$

for all $f \in C^{1}(\mathbb{R})$.

Proof of Proposition 3.1 From Russo and Vallois [29] we only need to show that

$$
[W, W]_{x}^{(S Q)}=|x|
$$

almost surely. That is, for each $t \geq 0$

$$
\left\|W^{\varepsilon}(x)-|x|\right\|_{L^{2}}^{2}=O\left(\varepsilon^{\alpha}\right)
$$

with some $\alpha>0$, as $\varepsilon \rightarrow 0$, by the above lemma, where

$$
W^{\varepsilon}(x)=\frac{1}{\varepsilon} \int_{I_{x}}\left(W_{y+\varepsilon}-W_{y}\right)^{2} d y .
$$

We have

$$
E\left|W^{\varepsilon}(x)-\right| x||^{2}=\frac{1}{\varepsilon^{2}} \int_{I_{x}} \int_{I_{x}} B_{\varepsilon}(y, z) d y d z
$$


for $x \in \mathbb{R}$ and $\varepsilon>0$, where

$$
\begin{aligned}
B_{\varepsilon}(y, z):= & E\left(\left(W_{y+\varepsilon}-W_{y}\right)^{2}-\varepsilon\right)\left(\left(W_{z+\varepsilon}-W_{z}\right)^{2}-\varepsilon\right) \\
= & E\left(W_{y+\varepsilon}-W_{y}\right)^{2}\left(W_{z+\varepsilon}-W_{z}\right)^{2}+\varepsilon^{2} \\
& -\varepsilon E\left(\left(W_{y+\varepsilon}-W_{y}\right)^{2}+\left(W_{z+\varepsilon}-W_{z}\right)^{2}\right) .
\end{aligned}
$$

Recall that

$$
\begin{aligned}
E\left[\left(W_{y+\varepsilon}-W_{y}\right)^{2}\right] & =\frac{1}{\sqrt{\pi}} \int_{0}^{t} \frac{1}{\sqrt{t-r}}\left(1-\exp \left\{-\frac{\varepsilon^{2}}{4(t-r)}\right\}\right) d r \\
& =\frac{1}{\sqrt{\pi}} \int_{0}^{t} \frac{1}{\sqrt{r}}\left(1-e^{-\frac{\varepsilon^{2}}{4 r}}\right) d r \\
& =\sqrt{\frac{2}{\pi}} \varepsilon \int_{\frac{\varepsilon}{\sqrt{2 t}}}^{\infty} \frac{1}{s^{2}}\left(1-e^{-\frac{s^{2}}{2}}\right) d s \equiv \phi_{t, y}(\varepsilon)+\varepsilon,
\end{aligned}
$$

where

$$
\phi_{t, y}(\varepsilon)=\sqrt{\frac{2}{\pi}} \varepsilon \int_{\frac{\varepsilon}{\sqrt{2 t}}}^{\infty} \frac{1}{s^{2}}\left(1-e^{-\frac{s^{2}}{2}}\right) d s-\varepsilon .
$$

Noting that

$$
\begin{aligned}
E[ & \left.\left(W_{y+\varepsilon}-W_{y}\right)^{2}\left(W_{z+\varepsilon}-W_{z}\right)^{2}\right] \\
= & E\left[\left(W_{y+\varepsilon}-W_{y}\right)^{2}\right] E\left[\left(W_{z+\varepsilon}-W_{z}\right)^{2}\right] \\
& +2\left(E\left[\left(W_{y+\varepsilon}-W_{y}\right)\left(W_{z+\varepsilon}-W_{z}\right)\right]\right)^{2}
\end{aligned}
$$

for all $\varepsilon>0$ and $y, z \in I_{x}$, we get

$$
B_{\varepsilon}(y, z)=\phi_{t, y}(\varepsilon) \phi_{t, y}(\varepsilon)+2\left(\mu_{y, z}\right)^{2},
$$

where $\mu_{y, z}:=E\left[\left(W_{y+\varepsilon}-W_{y}\right)\left(W_{z+\varepsilon}-W_{z}\right)\right]$.

Now, let us estimate the above function $\varepsilon \mapsto \phi_{t, y}(\varepsilon)$. We have

$$
\begin{aligned}
\phi_{t, y}(\varepsilon) & =\sqrt{\frac{2}{\pi}} \varepsilon\left(\int_{\frac{\varepsilon}{\sqrt{2 t}}}^{\infty} \frac{1}{s^{2}}\left(1-e^{-\frac{s^{2}}{2}}\right) d s-\sqrt{\frac{\pi}{2}}\right) \\
& =\sqrt{\frac{2}{\pi}} \varepsilon\left(\int_{\frac{\varepsilon}{\sqrt{2 t}}}^{\infty} \frac{1}{s^{2}}\left(1-e^{-\frac{s^{2}}{2}}\right) d s-\int_{0}^{\infty} \frac{1}{s^{2}}\left(1-e^{-\frac{s^{2}}{2}}\right) d s\right) \\
& =-\sqrt{\frac{2}{\pi}} \varepsilon \int_{0}^{\frac{\varepsilon}{\sqrt{2 t}}} \frac{1}{s^{2}}\left(1-e^{-\frac{s^{2}}{2}}\right) d s=O\left(\varepsilon^{2}\right) \quad(\varepsilon \rightarrow 0),
\end{aligned}
$$

which gives

$$
\frac{1}{\varepsilon^{2}} \int_{I_{x}} \int_{I_{x}}\left|\phi_{t, y}(\varepsilon) \phi_{t, z}(\varepsilon)\right| d y d z=O\left(\varepsilon^{2}\right) \quad(\varepsilon \rightarrow 0) .
$$

Combining this with Lemma 2.5, we complete the proof. 
Now, we discuss the existence of the PQC $[f(W), W]^{(S Q)}$. Consider the decomposition

$$
\begin{aligned}
I_{\varepsilon}^{1}(f, x, t) & =\frac{1}{\varepsilon} \int_{I_{x}} f\left(W_{y+\varepsilon}\right)\left(W_{y+\varepsilon}-W_{y}\right) d y-\frac{1}{\varepsilon} \int_{I_{x}} f\left(W_{y}\right)\left(W_{y+\varepsilon}-W_{y}\right) d y \\
& \equiv I_{\varepsilon}^{1,+}(f, x, t)-I_{\varepsilon}^{1,-}(f, x, t)
\end{aligned}
$$

for $\varepsilon>0$, and define the set

$$
\mathscr{H}_{t}=\left\{f: \text { Borel functions on } \mathbb{R} \text { such that }\|f\|_{\mathscr{H}_{t}}<\infty\right\},
$$

where

$$
\|f\|_{\mathscr{H}_{t}}^{2}:=\frac{1}{\sqrt[4]{4 \pi t}} \int_{\mathbb{R}}|f(z)|^{2}\left(\sqrt{t}+z^{2}\right) e^{-\frac{\sqrt{\pi} z^{2}}{2 \sqrt{t}}} d z .
$$

Then $\mathscr{H}_{t}=L^{2}\left(\mathbb{R}, \mu_{t}(d z)\right)$ with

$$
\mu_{t}(d z)=\left(\frac{1}{\sqrt[4]{4 \pi t}}\left(\sqrt{t}+z^{2}\right) e^{-\frac{\sqrt{\pi} z^{2}}{2 \sqrt{t}}}\right) d z
$$

and $\mu_{t}(\mathbb{R})=C|x|<\infty$, which implies that the set $\mathscr{E}$ of elementary functions of the form

$$
f_{\triangle}(z)=\sum_{i} f_{i} 1_{\left(x_{i-1}, x_{i}\right]}(z)
$$

is dense in $\mathscr{H}_{t}$, where $f_{i} \in \mathbb{R}$ and $\left\{x_{i}, 0 \leq i \leq l\right\}$ is a finite sequence of real numbers such that $x_{i}<x_{i+1}$. Moreover, $\mathscr{H}_{t}$ includes all Borel functions $f$ satisfying the condition

$$
|f(z)| \leq C e^{\beta z^{2}}, \quad z \in \mathbb{R}
$$

with $0 \leq \beta<\frac{\sqrt{\pi}}{4 \sqrt{t}}$.

Theorem 3.1 Let $f \in \mathscr{H}_{t}$. Then the PQC $[f(W), W]^{(S Q)}$ exists in $L^{2}(\Omega)$ and

$$
E\left|[f(W), W]_{x}^{(S Q)}\right|^{2} \leq C M\|f\|_{\mathscr{H}_{t}}^{2}
$$

for all $x \in[-M, M]$.

In order to prove the theorem it is enough to prove the following two statements with $f \in \mathscr{H}_{t}$ :

(1) For any $\varepsilon>0$ and $x \in[-M, M], I_{\varepsilon}^{1, \pm}(f, x, \cdot) \in L^{2}(\Omega)$. That is,

$$
\begin{aligned}
& E\left|I_{\varepsilon}^{1,-}(f, x, \cdot)\right|^{2} \leq C M\|f\|_{\mathscr{H}_{t}}^{2}, \\
& E\left|I_{\varepsilon}^{1,+}(f, x, \cdot)\right|^{2} \leq C M\|f\|_{\mathscr{H}_{t}}^{2} .
\end{aligned}
$$

(2) $I_{\varepsilon}^{1,-}(f, x, t)$ and $I_{\varepsilon}^{1,+}(f, x, t)$ are two Cauchy sequences in $L^{2}(\Omega)$ for all $t>0$ and $x \in[-M, M]$. That is,

$$
E\left|I_{\varepsilon_{1}}^{1,-}(f, x, t)-I_{\varepsilon_{2}}^{1,-}(f, x, t)\right|^{2} \longrightarrow 0
$$


and

$$
E\left|I_{\varepsilon_{1}}^{1,+}(f, x, t)-I_{\varepsilon_{2}}^{1,+}(f, x, t)\right|^{2} \longrightarrow 0
$$

for all $x \in \mathbb{R}$, as $\varepsilon_{1}, \varepsilon_{2} \downarrow 0$.

We split the proof of the two statements into two parts.

Proof of statement (1) Let $x \in[-M, M]$. We have

$$
E\left|I_{\varepsilon}^{1,-}(f, x, \cdot)\right|^{2}=\frac{1}{\varepsilon^{2}} \int_{I_{x}} \int_{I_{x}} d y d y^{\prime} E\left[f\left(W_{y}\right) f\left(W_{y^{\prime}}\right)\left(W_{y+\varepsilon}-W_{y}\right)\left(W_{y^{\prime}+\varepsilon}-W_{y^{\prime}}\right)\right]
$$

for all $\varepsilon>0$. Now, let us estimate the expression

$$
\Phi_{\varepsilon_{1}, \varepsilon_{2}}\left(y, y^{\prime}\right):=E\left[f\left(W_{y}\right) f\left(W_{y^{\prime}}\right)\left(W_{y+\varepsilon_{1}}-W_{y}\right)\left(W_{y^{\prime}+\varepsilon_{2}}-W_{y^{\prime}}\right)\right]
$$

for all $\varepsilon_{1}, \varepsilon_{2}>0$ and $y, y^{\prime} \in I_{x}$. To estimate the above expression, it is enough to assume that $f \in \mathscr{E}$ by denseness, and moreover, by approximating we can assume that $f$ is an infinitely differentiable function with compact support. It follows from the duality relationship (2.1) that

$$
\begin{aligned}
\Phi_{\varepsilon_{1}, \varepsilon_{2}}\left(y, y^{\prime}\right)= & E\left[f\left(W_{y}\right) f\left(W_{y^{\prime}}\right)\left(W_{y+\varepsilon_{1}}-W_{y}\right) \int_{y^{\prime}}^{y^{\prime}+\varepsilon_{2}} \delta W_{l}\right] \\
= & E\left[W_{y}\left(W_{y^{\prime}+\varepsilon_{2}}-W_{y^{\prime}}\right)\right] E\left[f^{\prime}\left(W_{y}\right) f\left(W_{y^{\prime}}\right)\left(W_{y+\varepsilon_{1}}-W_{y}\right)\right] \\
& +E\left[W_{y^{\prime}}\left(W_{y^{\prime}+\varepsilon_{2}}-W_{y^{\prime}}\right)\right] E\left[f\left(W_{y}\right) f^{\prime}\left(W_{y^{\prime}}\right)\left(W_{y+\varepsilon_{1}}-W_{y}\right)\right] \\
& +E\left[\left(W_{y+\varepsilon_{1}}-W_{y}\right)\left(W_{y^{\prime}+\varepsilon_{2}}-W_{y^{\prime}}\right)\right] E\left[f\left(W_{y}\right) f\left(W_{y^{\prime}}\right)\right] \\
= & E\left[W_{y}\left(W_{y^{\prime}+\varepsilon_{2}}-W_{y^{\prime}}\right)\right] E\left[W_{y}\left(W_{y+\varepsilon_{2}}-W_{y}\right)\right] E\left[f^{\prime \prime}\left(W_{y}\right) f\left(W_{y^{\prime}}\right)\right] \\
& +E\left[W_{y}\left(W_{y^{\prime}+\varepsilon_{2}}-W_{y^{\prime}}\right)\right] E\left[W_{y^{\prime}}\left(W_{y+\varepsilon_{2}}-W_{y}\right)\right] E\left[f^{\prime}\left(W_{y}\right) f^{\prime}\left(W_{y^{\prime}}\right)\right] \\
& +E\left[W_{y^{\prime}}\left(W_{y^{\prime}+\varepsilon_{2}}-W_{y^{\prime}}\right)\right] E\left[W_{y}\left(W_{y+\varepsilon_{1}}-W_{y}\right)\right] E\left[f^{\prime}\left(W_{y}\right) f^{\prime}\left(W_{y^{\prime}}\right)\right] \\
& +E\left[W_{y^{\prime}}\left(W_{y^{\prime}+\varepsilon_{2}}-W_{y^{\prime}}\right)\right] E\left[W_{y^{\prime}}\left(W_{y+\varepsilon_{1}}-W_{y}\right)\right] E\left[f\left(W_{y}\right) f^{\prime \prime}\left(W_{y^{\prime}}\right)\right] \\
& +E\left[\left(W_{y+\varepsilon_{1}}-W_{y}\right)\left(W_{y^{\prime}+\varepsilon_{2}}-W_{y^{\prime}}\right)\right] E\left[f\left(W_{y}\right) f\left(W_{y^{\prime}}\right)\right] \\
\equiv & \sum_{j=1}^{5} \Psi_{j}\left(y, y^{\prime}, \varepsilon_{1}, \varepsilon_{2}\right)
\end{aligned}
$$

for all $y, y^{\prime} \in I_{x}$ and $\varepsilon_{1}, \varepsilon_{2}>0$. In order to end the proof we claim the

$$
\Lambda_{j}:=\frac{1}{\varepsilon^{2}} \int_{I_{x}} \int_{I_{x}} \Psi_{j}\left(y, y^{\prime}, \varepsilon, \varepsilon\right) d y d y^{\prime}, \quad j=1,2,3,4,5,
$$

for all $\varepsilon>0$ to be small enough.

For $j=5$, from the fact

$$
\left|E\left[\left(W_{y+\varepsilon}-W_{y}\right)\left(W_{y^{\prime}+\varepsilon}-W_{y^{\prime}}\right)\right]\right| \leq \varepsilon
$$


for $0<\left|y-y^{\prime}\right| \leq \varepsilon$, we have

$$
\begin{aligned}
& \frac{1}{\varepsilon^{2}} \int_{\substack{\left|y-y^{\prime}\right| \leq \varepsilon \\
y, y^{\prime} \in I_{x}}}\left|\Psi_{5}\left(y, y^{\prime}, \varepsilon, \varepsilon\right)\right| d y d y^{\prime} \\
& \quad \leq \frac{1}{\varepsilon} \int_{\substack{\left|y-y^{\prime}\right| \leq \varepsilon \\
y, y^{\prime} \in I_{x}}} E\left|f\left(W_{y}\right) f\left(W_{y^{\prime}}\right)\right| d y d y^{\prime} \\
& \quad \leq \frac{1}{2 \varepsilon} \int_{\substack{\left|y-y^{\prime}\right| \leq \varepsilon \\
y, y^{\prime} \in I_{x}}} E\left[\left|f\left(W_{y}\right)\right|^{2}+\left|f\left(W_{y^{\prime}}\right)\right|^{2}\right] d y d y^{\prime} \\
& \quad \leq \frac{1}{\varepsilon} \int_{\substack{\left|y-y^{\prime}\right| \leq \varepsilon \\
y, y^{\prime} \in I_{x}}} E\left|f\left(W_{y}\right)\right|^{2} d y d y^{\prime} \\
& \quad \leq \int_{I_{x}} E\left|f\left(W_{y}\right)\right|^{2} d y=\|f\|_{\mathscr{H}_{t}}^{2}
\end{aligned}
$$

for all $\varepsilon>0$. Moreover, for $\left|y-y^{\prime}\right|>\varepsilon$ we have

$$
\left|E\left[\left(W_{y+\varepsilon}-W_{y}\right)\left(W_{y^{\prime}+\varepsilon}-W_{y^{\prime}}\right)\right]\right| \leq \frac{1}{4 \sqrt{t \pi}} \varepsilon^{2} e^{-\frac{\left|y-y^{\prime}-\varepsilon\right|^{2}}{4 t}}
$$

by (2.8), which implies that

$$
\begin{aligned}
& \frac{1}{\varepsilon^{2}} \int_{\substack{\left\{\left|y-y^{\prime}\right|>\varepsilon^{\prime} \\
y, y^{\prime} \in I_{x}\right.}}\left|\Psi_{5}\left(y, y^{\prime}, \varepsilon, \varepsilon\right)\right| d y d y^{\prime} \\
& \quad \leq \frac{1}{4 \sqrt{t \pi}} \int_{\substack{\left\{y-y^{\prime} \mid>\varepsilon \\
y, y^{\prime} \in I_{x}\right.}} E\left|f\left(W_{y}\right) f\left(W_{y^{\prime}}\right)\right| e^{-\frac{\left|y-y^{\prime}-\varepsilon\right|^{2}}{4 t}} d y d y^{\prime} \\
& \quad \leq \frac{1}{8 \sqrt{t \pi}} \int_{\substack{\left\{\left|y-y^{\prime}\right|>\varepsilon \\
y, y^{\prime} \in I_{x}\right.}} E\left[\left|f\left(W_{y}\right)\right|^{2}+\left|f\left(W_{y^{\prime}}\right)\right|^{2}\right] e^{-\frac{\left.\left|y-y^{\prime}-\varepsilon\right|\right|^{2}}{4 t}} d y d y^{\prime} \\
& \quad \leq \frac{1}{4 \sqrt{t \pi}} \int_{\substack{\left\{y-y^{\prime} \mid>\varepsilon^{\prime} \\
y, y^{\prime} \in I_{x}\right.}} E\left|f\left(W_{y}\right)\right|^{2} e^{-\frac{\left|y-y^{\prime}-\varepsilon\right|^{2}}{4 t}} d y d y^{\prime} \\
& \quad \leq \frac{1}{2} \int_{I_{x}} E\left|f\left(W_{y}\right)\right|^{2} d y \int_{-\infty}^{\infty} \frac{1}{\sqrt{2 \pi(2 t)}} e^{-\frac{\left|y-y^{\prime}-\varepsilon\right|^{2}}{4 t}} d y^{\prime} \\
& \quad=\frac{1}{2} \int_{I_{x}} E\left|f\left(W_{y}\right)\right|^{2} d y=\frac{1}{2}\|f\|_{\mathscr{H}}^{2}
\end{aligned}
$$

for all $\varepsilon>0$. This shows that

$$
\Lambda_{5}=\frac{1}{\varepsilon^{2}}\left|\int_{I_{x}} \int_{I_{x}} \Psi_{5}\left(y, y^{\prime}, \varepsilon, \varepsilon\right) d y d y^{\prime}\right| \leq\|f\|_{\mathscr{H}_{t}}^{2}
$$

for all $\varepsilon>0$.

Next, let us estimate $\sum_{j=1}^{4} \Lambda_{j}$. We have

$$
\begin{aligned}
E\left[f^{\prime \prime}\left(W_{y}\right) f\left(W_{y^{\prime}}\right)\right] & =\int_{\mathbb{R}^{2}} f(x) f\left(x^{\prime}\right) \frac{\partial^{2}}{\partial x^{2}} \varphi\left(x, x^{\prime}\right) d x d x^{\prime} \\
& =\int_{\mathbb{R}^{2}} f(x) f\left(x^{\prime}\right)\left\{\frac{1}{\rho^{4}}\left(\sigma_{t, y^{\prime}}^{2} x-\mu_{t, y, y^{\prime}} x^{\prime}\right)^{2}-\frac{\sigma_{t, y^{\prime}}^{2}}{\rho^{2}}\right\} \varphi\left(x, x^{\prime}\right) d x d x^{\prime}
\end{aligned}
$$


and

$$
\begin{aligned}
E & {\left[f^{\prime}\left(W_{y}\right) f^{\prime}\left(W_{y^{\prime}}\right)\right] } \\
& =\int_{\mathbb{R}^{2}} f(x) f\left(x^{\prime}\right) \frac{\partial^{2}}{\partial x \partial x^{\prime}} \varphi\left(x, x^{\prime}\right) d x d x^{\prime} \\
& =\int_{\mathbb{R}^{2}} f(x) f\left(x^{\prime}\right)\left\{\frac{1}{\rho^{4}}\left(\sigma_{t, y}^{2} x^{\prime}-\mu_{t, y, y^{\prime}} x\right)\left(\sigma_{t, y^{\prime}}^{2} x-\mu_{t, y, y^{\prime}} x^{\prime}\right)+\frac{\mu_{t, y, y^{\prime}}}{\rho^{2}}\right\} \varphi\left(x, x^{\prime}\right) d x d x^{\prime},
\end{aligned}
$$

where $\rho^{2}=\sigma_{t, y}^{2} \sigma_{t, y^{\prime}}^{2}-\mu_{t, y, y^{\prime}}^{2}$ and $\varphi(x, y)$ is the density function of $\left(W_{y}, W_{y^{\prime}}\right)$, that is,

$$
\varphi\left(x, x^{\prime}\right)=\frac{1}{2 \pi \rho} \exp \left\{-\frac{1}{2 \rho^{2}}\left(\sigma_{t, y^{\prime}}^{2} x^{2}-2 \mu_{t, y, y^{\prime}} x x^{\prime}+\sigma_{t, y}^{2} x^{\prime 2}\right)\right\} .
$$

Combining this with the identity

$$
\begin{aligned}
& \left(\sigma_{t, y^{2}}^{2} x^{\prime}-\mu_{t, y, y^{\prime}} x\right)\left(\sigma_{t, y^{\prime}}^{2} x-\mu_{t, y, y^{\prime}} x^{\prime}\right) \\
& \quad=\rho^{2} x^{\prime}\left(x-\frac{\mu_{t, y, y^{\prime}}}{\sigma_{t, y^{\prime}}^{2}} x^{\prime}\right)-\mu_{t, y, y^{\prime}} \sigma_{t, y^{\prime}}^{2}\left(x-\frac{\mu_{t, y, y^{\prime}}}{\sigma_{t, y^{\prime}}^{2}} x^{\prime}\right)^{2},
\end{aligned}
$$

we get

$$
\begin{aligned}
& E\left[f^{\prime \prime}\left(W_{y}\right) f\left(W_{y^{\prime}}\right)\right]+E\left[f^{\prime}\left(W_{y}\right) f^{\prime}\left(W_{y^{\prime}}\right)\right] \\
& =\frac{\mu_{t, y, y^{\prime}}-\sigma_{t, y^{\prime}}^{2}}{\rho^{2}} \int_{\mathbb{R}} \int_{\mathbb{R}} f(x) f\left(x^{\prime}\right) \varphi\left(x, x^{\prime}\right) d x d x^{\prime} \\
& \quad+\frac{1}{\rho^{2}} \int_{\mathbb{R}} \int_{\mathbb{R}} f(x) f\left(x^{\prime}\right) x^{\prime}\left(x-\frac{\mu_{t, y, y^{\prime}}}{\sigma_{t, y^{\prime}}^{2}} x^{\prime}\right) \varphi\left(x, x^{\prime}\right) d x d x^{\prime} \\
& \quad+\frac{\sigma_{t, y^{\prime}}^{2}}{\rho^{4}}\left(\sigma_{t, y^{\prime}}^{2}-\mu_{t, y, y^{\prime}}\right) \int_{\mathbb{R}} \int_{\mathbb{R}} f(x) f\left(x^{\prime}\right)\left(x-\frac{\mu_{t, y, y^{\prime}}}{\sigma_{t, y^{\prime}}^{2}} x^{\prime}\right)^{2} \varphi\left(x, x^{\prime}\right) d x d x^{\prime} \\
& \equiv \Upsilon_{1}+\Upsilon_{2}+\Upsilon_{3} .
\end{aligned}
$$

A straightforward calculation shows that

$$
\begin{aligned}
& \int_{\mathbb{R}} \int_{\mathbb{R}}\left|f\left(x^{\prime}\right)\right|^{2}\left(x-\frac{\mu_{t, y, y^{\prime}}}{\sigma_{t, y^{\prime}}^{2}} x^{\prime}\right)^{2 m} \varphi\left(x, x^{\prime}\right) d x d x^{\prime} \\
& =C_{m}\left(\frac{\rho^{2}}{\sigma_{t, y^{\prime}}^{2}}\right)^{m} \int_{\mathbb{R}}\left|f\left(x^{\prime}\right)\right|^{2} \frac{1}{\sqrt{2 \pi} \sigma_{t, y^{\prime}}} e^{-\frac{x^{\prime 2}}{2 \sigma_{t, y^{\prime}}^{2}}} d x^{\prime} \\
& \leq C_{m}\left(\frac{\rho^{2}}{\sqrt{t}}\right)^{m} \int_{\mathbb{R}}|f(x)|^{2} \frac{1}{\sqrt[4]{t}} e^{-\frac{\sqrt{\pi} x^{2}}{2 \sqrt{t}}} d x
\end{aligned}
$$

for all $m \geq 1$ and

$$
\int_{\mathbb{R}} \int_{\mathbb{R}}\left|f(x) x^{\prime}\right|^{2} \varphi\left(x, x^{\prime}\right) d x d x^{\prime}
$$




$$
\begin{aligned}
& =\int_{\mathbb{R}}|f(x)|^{2} \frac{1}{\sqrt{2 \pi} \sigma_{t, y}} e^{-\frac{x^{2}}{2 \sigma_{t, y}^{2}}} d x \int_{\mathbb{R}}\left|x^{\prime}\right|^{2} \frac{\sigma_{t, y}}{\sqrt{2 \pi} \rho} e^{-\frac{\sigma_{t, y}^{2}}{2 \rho^{2}}\left(x^{\prime}-\frac{\mu_{t, y, y^{\prime}}}{\sigma_{t, y}^{2}} x\right)^{2}} d x^{\prime} \\
& =\int_{\mathbb{R}}|f(x)|^{2} \frac{1}{\sqrt{2 \pi} \sigma_{t, y}} e^{-\frac{x^{2}}{2 \sigma_{t, y}^{2}}} d x\left(\frac{\rho^{2}}{\sigma_{t, y}^{2}}+\frac{\mu_{t, y, y^{\prime}}^{2}}{\sigma_{t, y}^{4}} x^{2}\right) \\
& \leq C \frac{1}{\sqrt[4]{\pi t}} \int_{\mathbb{R}}|f(x)|^{2} e^{-\frac{\sqrt{\pi} x^{2}}{2 \sqrt{t}}}\left(\sqrt{t}+x^{2}\right) d x
\end{aligned}
$$

since $\sigma_{t, y}^{2}=\sigma_{t, y^{\prime}}^{2}=\sqrt{\frac{t}{\pi}}$. It follows that

$$
\begin{aligned}
\left|\Upsilon_{1}\right| & \leq \frac{1}{\rho^{2}}\left(\int_{\mathbb{R}^{2}}\left|f(x) x^{\prime}\right|^{2} \varphi\left(x, x^{\prime}\right) d x d x^{\prime} \int_{\mathbb{R}^{2}}\left|f\left(x^{\prime}\right)\right|^{2}\left|x-\frac{\mu_{t, y, y^{\prime}}}{\sigma_{t, y^{\prime}}^{2}} x^{\prime}\right|^{2} \varphi\left(x, x^{\prime}\right) d x d x^{\prime}\right)^{\frac{1}{2}} \\
& \leq C \frac{1}{\rho \sqrt[4]{t}} \int_{\mathbb{R}}|f(x)|^{2} \frac{1}{\sqrt[4]{t}} e^{-\frac{\sqrt{\pi} x^{2}}{2 \sqrt{t}}}\left(\sqrt{t}+x^{2}\right) d x
\end{aligned}
$$

and

$$
\begin{aligned}
\left|\Upsilon_{3}\right| \leq & \frac{\sigma_{t, y^{\prime}}^{2}}{\rho^{4}}\left|\sigma_{t, y^{\prime}}^{2}-\mu_{t, y, y^{\prime}}\right| \\
& \times\left(\int_{\mathbb{R}^{2}}|f(x)|^{2} \varphi\left(x, x^{\prime}\right) d x d x^{\prime} \int_{\mathbb{R}^{2}}\left|f\left(x^{\prime}\right)\right|^{2}\left(x-\frac{\mu_{t, y, y^{\prime}}}{\sigma_{t, y^{\prime}}^{2}} x^{\prime}\right)^{4} \varphi\left(x, x^{\prime}\right) d x d x^{\prime}\right)^{1 / 2} \\
\leq & \frac{C\left|\sigma_{t, y^{\prime}}^{2}-\mu_{t, y, y^{\prime}}\right|}{\rho^{2}} \int_{\mathbb{R}}|f(x)|^{2} \frac{1}{\sqrt[4]{t}} e^{-\frac{\sqrt{\pi} x^{2}}{2 \sqrt{t}}} d x .
\end{aligned}
$$

Thus, we get the estimate

$$
\begin{aligned}
\mid E & {\left[f^{\prime \prime}\left(W_{y}\right) f\left(W_{y^{\prime}}\right)\right]+E\left[f^{\prime}\left(W_{y}\right) f^{\prime}\left(W_{y^{\prime}}\right)\right] \mid } \\
& \leq\left|\Upsilon_{1}\right|+\left|\Upsilon_{2}\right|+\left|\Upsilon_{3}\right| \\
& \leq \int_{\mathbb{R}}|f(x)|^{2} \frac{1}{\sqrt[4]{t}} e^{-\frac{\sqrt{\pi} x^{2}}{2 \sqrt{t}}}\left(\sqrt{t}+x^{2}\right) d x
\end{aligned}
$$

and

$$
\left|E\left[f^{\prime \prime}\left(W_{y}\right) f\left(W_{y^{\prime}}\right)\right]\right| \leq \frac{C}{\left|y-y^{\prime}\right|} \int_{\mathbb{R}}|f(x)|^{2} \frac{1}{\sqrt[4]{t}} e^{-\frac{\sqrt{\pi} x^{2}}{2 \sqrt{t}}} d x
$$

by Lemma 2.7 and Lemma 2.3. Now, we can estimate $\sum_{j=1}^{4} \Lambda_{j}$. We have

$$
\begin{aligned}
\sum_{j=1}^{4} \Psi_{j}\left(y, y^{\prime}, \varepsilon, \varepsilon\right) \\
=E\left[W_{y}\left(W_{y^{\prime}+\varepsilon}-W_{y^{\prime}}\right)\right] E\left[\left(W_{y}-W_{y^{\prime}}\right)\left(W_{y+\varepsilon}-W_{y}\right)\right] E\left[f^{\prime \prime}\left(W_{y}\right) f\left(W_{y^{\prime}}\right)\right] \\
\quad+E\left[W_{y}\left(W_{y^{\prime}+\varepsilon}-W_{y^{\prime}}\right)\right] E\left[W_{y^{\prime}}\left(W_{y+\varepsilon}-W_{y}\right)\right] \\
\quad \times\left(E\left[f^{\prime}\left(W_{y}\right) f^{\prime}\left(W_{y^{\prime}}\right)\right]+E\left[f^{\prime \prime}\left(W_{y}\right) f\left(W_{y^{\prime}}\right)\right]\right)
\end{aligned}
$$




$$
\begin{aligned}
& +E\left[W_{y^{\prime}}\left(W_{y^{\prime}+\varepsilon}-W_{y^{\prime}}\right)\right] E\left[W_{y}\left(W_{y+\varepsilon}-W_{y}\right)\right] \\
& \times\left(E\left[f^{\prime}\left(W_{y}\right) f^{\prime}\left(W_{y^{\prime}}\right)\right]+E\left[f\left(W_{y}\right) f^{\prime \prime}\left(W_{y^{\prime}}\right)\right]\right) \\
& +E\left[W_{y^{\prime}}\left(W_{y^{\prime}+\varepsilon}-W_{y^{\prime}}\right)\right] E\left[\left(W_{y^{\prime}}-W_{y}\right)\left(W_{y+\varepsilon}-W_{y}\right)\right] E\left[f\left(W_{y}\right) f^{\prime \prime}\left(W_{y^{\prime}}\right)\right] .
\end{aligned}
$$

Combining this with (3.9), (3.10), Lemma 2.3 and Lemma 2.5, we get

$$
\left|\sum_{j=1}^{4} \Lambda_{j}\right| \leq \frac{1}{\varepsilon^{2}} \int_{I_{x}} \int_{I_{x}}\left|\sum_{j=1}^{4} \Psi_{j}\left(y, y^{\prime}, \varepsilon, \varepsilon\right)\right| d y d y^{\prime} \leq C M\|f\|_{\mathscr{H}_{t}}^{2}
$$

for all $\varepsilon>0$ and $x \in \mathbb{R}$. This shows that

$$
E\left|I_{\varepsilon}^{1,-}(f, x, \cdot)\right|^{2} \leq C M\|f\|_{\mathscr{H}_{t}}^{2}
$$

Similarly, one can show the estimate

$$
E\left|I_{\varepsilon}^{1,+}(f, x, \cdot)\right|^{2} \leq C M\|f\|_{\mathscr{H}_{t}}^{2},
$$

and the first statement follows.

Proof of statement (2) Given $x \in[-M, M]$. Without loss of generality we assume that $\varepsilon_{1}>\varepsilon_{2}$. We prove only the first convergence and similarly one can prove the second convergence. We have

$$
\begin{aligned}
& E\left|I_{\varepsilon_{1}}^{1,-}(f, x, t)-I_{\varepsilon_{2}}^{1,-}(f, x, t)\right|^{2} \\
&=\frac{1}{\varepsilon_{1}^{2}} \int_{I_{x}} \int_{I_{x}} E f\left(W_{y}\right) f\left(W_{y^{\prime}}\right)\left(W_{y+\varepsilon_{1}}-W_{y}\right)\left(W_{y^{\prime}+\varepsilon_{1}}-W_{y^{\prime}}\right) d y d y^{\prime} \\
& \quad-2 \frac{1}{\varepsilon_{1} \varepsilon_{2}} \int_{I_{x}} \int_{I_{x}} E f\left(W_{y}\right) f\left(W_{y^{\prime}}\right)\left(W_{y+\varepsilon_{1}}-W_{y}\right)\left(W_{y^{\prime}+\varepsilon_{2}}-W_{y^{\prime}}\right) d y d y^{\prime} \\
& \quad+\frac{1}{\varepsilon_{2}^{2}} \int_{I_{x}} \int_{I_{x}} E f\left(W_{y}\right) f\left(W_{y^{\prime}}\right)\left(W_{y+\varepsilon_{2}}-W_{y}\right)\left(W_{y^{\prime}+\varepsilon_{2}}-W_{y^{\prime}}\right) d y d y^{\prime} \\
& \equiv \frac{1}{\varepsilon_{1}^{2} \varepsilon_{2}} \int_{I_{x}} \int_{I_{x}}\left\{\varepsilon_{2} \Phi_{y, y^{\prime}}\left(1, \varepsilon_{1}\right)-\varepsilon_{1} \Phi_{y, y^{\prime}}\left(2, \varepsilon_{1}, \varepsilon_{2}\right)\right\} d y d y^{\prime} \\
&+\frac{1}{\varepsilon_{1} \varepsilon_{2}^{2}} \int_{I_{x}} \int_{I_{x}}\left\{\varepsilon_{1} \Phi_{y, y^{\prime}}\left(1, \varepsilon_{2}\right)-\varepsilon_{2} \Phi_{y, y^{\prime}}\left(2, \varepsilon_{1}, \varepsilon_{2}\right)\right\} d y d y^{\prime},
\end{aligned}
$$

for all $\varepsilon_{1}, \varepsilon_{2}>0$, where

$$
\Phi_{y, y^{\prime}}(1, \varepsilon)=E\left[f\left(W_{y}\right) f\left(W_{y^{\prime}}\right)\left(W_{y+\varepsilon}-W_{y}\right)\left(W_{y^{\prime}+\varepsilon}-W_{y^{\prime}}\right)\right]
$$

and

$$
\Phi_{y, y^{\prime}}\left(2, \varepsilon_{1}, \varepsilon_{2}\right)=E\left[f\left(W_{y}\right) f\left(W_{y^{\prime}}\right)\left(W_{y+\varepsilon_{1}}-W_{y}\right)\left(W_{y^{\prime}+\varepsilon_{2}}-W_{y^{\prime}}\right)\right] .
$$

To end the proof, it is enough to assume that $f \in \mathscr{E}$ by denseness, and moreover, by approximating we can assume that $f$ is an infinitely differentiable function with compact 
support. It follows from (3.8) that

$$
\Phi_{y, y^{\prime}}(1, \varepsilon)=\sum_{j=1}^{5} \Psi_{j}\left(y, y^{\prime}, \varepsilon, \varepsilon\right), \Phi_{y, y^{\prime}}\left(2, \varepsilon_{1}, \varepsilon_{2}\right)=\sum_{j=1}^{5} \Psi_{j}\left(y, y^{\prime}, \varepsilon_{1}, \varepsilon_{2}\right),
$$

which gives

$$
\begin{aligned}
\varepsilon_{j} \Phi_{y, y^{\prime}} & \left(1, \varepsilon_{i}\right)-\varepsilon_{i} \Phi_{y, y^{\prime}}\left(2, \varepsilon_{1}, \varepsilon_{2}\right) \\
= & A_{y, y^{\prime}}\left(1, \varepsilon_{i}, j\right) E\left[f^{\prime \prime}\left(W_{y}\right) f\left(W_{y^{\prime}}\right)\right]+A_{y, y^{\prime}}\left(2-1, \varepsilon_{i}, j\right) E\left[f^{\prime}\left(W_{y}\right) f^{\prime}\left(W_{y^{\prime}}\right)\right] \\
& +A_{y, y^{\prime}}\left(3, \varepsilon_{i}, j\right) E\left[f\left(W_{y}\right) f^{\prime \prime}\left(W_{y^{\prime}}\right)\right]+A_{y, y^{\prime}}\left(2-2, \varepsilon_{i}, j\right) E\left[f^{\prime}\left(W_{y}\right) f^{\prime}\left(W_{y^{\prime}}\right)\right] \\
& +A_{y, y^{\prime}}(4, \varepsilon, j) E\left[f\left(W_{y}\right) f\left(W_{y^{\prime}}\right]\right.
\end{aligned}
$$

with $i, j \in\{1,2\}$ and $i \neq j$, where

$$
\begin{aligned}
A_{y, y^{\prime}} & (1, \varepsilon, j) \\
:= & \varepsilon_{j} E\left[W_{y}\left(W_{y^{\prime}+\varepsilon}-W_{y^{\prime}}\right)\right] E\left[W_{y}\left(W_{y+\varepsilon}-W_{y}\right)\right] \\
& -\varepsilon E\left[W_{y}\left(W_{y^{\prime}+\varepsilon_{2}}-W_{y^{\prime}}\right)\right] E\left[W_{y}\left(W_{y+\varepsilon_{1}}-W_{y}\right)\right] \\
A_{y, y^{\prime}} & (2-1, \varepsilon, j) \\
:= & \varepsilon_{j} E\left[W_{y}\left(W_{y^{\prime}+\varepsilon}-W_{y^{\prime}}\right)\right] E\left[W_{y^{\prime}}\left(W_{y+\varepsilon}-W_{y}\right)\right] \\
& -\varepsilon E\left[W_{y}\left(W_{y^{\prime}+\varepsilon_{2}}-W_{y^{\prime}}\right)\right] E\left[W_{y^{\prime}}\left(W_{y+\varepsilon_{1}}-W_{y}\right)\right] \\
A_{y, y^{\prime}} & (2-2, \varepsilon, j) \\
:= & \varepsilon_{j} E\left[W_{y^{\prime}}\left(W_{y^{\prime}+\varepsilon}-W_{y^{\prime}}\right)\right] E\left[W_{y}\left(W_{y+\varepsilon}-W_{y}\right)\right] \\
& -\varepsilon E\left[W_{y^{\prime}}\left(W_{y^{\prime}+\varepsilon_{2}}-W_{y^{\prime}}\right)\right] E\left[W_{y}\left(W_{y+\varepsilon_{1}}-W_{y}\right)\right] \\
A_{y, y^{\prime}} & (3, \varepsilon, j) \\
:= & \varepsilon_{j} E\left[W_{y^{\prime}}\left(W_{y^{\prime}+\varepsilon}-W_{y^{\prime}}\right)\right] E\left[W_{y^{\prime}}\left(W_{y+\varepsilon}-W_{y}\right)\right] \\
& -\varepsilon E\left[W_{y^{\prime}}\left(W_{y^{\prime}+\varepsilon_{2}}-W_{y^{\prime}}\right)\right] E\left[W_{y^{\prime}}\left(W_{y+\varepsilon_{1}}-W_{y}\right)\right] \\
A_{y, y^{\prime}}(4, \varepsilon, j) & \\
:= & \varepsilon_{j} E\left[\left(W_{y+\varepsilon}-W_{y}\right)\left(W_{y^{\prime}+\varepsilon}-W_{y^{\prime}}\right)\right]-\varepsilon E\left[\left(W_{y+\varepsilon_{1}}-W_{y}\right)\left(W_{y^{\prime}+\varepsilon_{2}}-W_{y^{\prime}}\right)\right]
\end{aligned}
$$

for all $\varepsilon_{1}, \varepsilon_{2}>0$ and $y, y^{\prime} \in \mathbb{R}$. Now, we claim that the following convergences hold:

$$
\frac{1}{\varepsilon_{i}^{2} \varepsilon_{j}} \int_{0}^{t} \int_{0}^{t}\left\{\varepsilon_{j} \Phi_{y, y^{\prime}}\left(1, \varepsilon_{i}\right)-\varepsilon_{i} \Phi_{y, y^{\prime}}\left(2, \varepsilon_{1}, \varepsilon_{2}\right)\right\} d s d r \longrightarrow 0
$$

with $i, j \in\{1,2\}$ and $i \neq j$, as $\varepsilon_{1}, \varepsilon_{2} \rightarrow 0$. We decompose

$$
\begin{aligned}
\varepsilon_{j} \Phi_{y, y^{\prime}} & \left(1, \varepsilon_{i}\right)-\varepsilon_{i} \Phi_{y, y^{\prime}}\left(2, \varepsilon_{1}, \varepsilon_{2}\right) \\
= & \left\{A_{y, y^{\prime}}\left(1, \varepsilon_{i}, j\right)-A_{y, y^{\prime}}\left(2-1, \varepsilon_{i}, j\right)\right\} E\left[f^{\prime \prime}\left(W_{y}\right) f\left(W_{y^{\prime}}\right)\right] \\
& +A_{y, y^{\prime}}\left(2-1, \varepsilon_{i}, j\right)\left\{E\left[f^{\prime}\left(W_{y}\right) f^{\prime}\left(W_{y^{\prime}}\right)\right]+E\left[f^{\prime \prime}\left(W_{y}\right) f\left(W_{y^{\prime}}\right)\right]\right\}
\end{aligned}
$$




$$
\begin{aligned}
& +\left\{A_{y, y^{\prime}}\left(3, \varepsilon_{i}, j\right)-A_{y, y^{\prime}}\left(2-2, \varepsilon_{i}, j\right)\right\} E\left[f\left(W_{y}\right) f^{\prime \prime}\left(W_{y^{\prime}}\right)\right] \\
& +A_{y, y^{\prime}}\left(2-2, \varepsilon_{i}, j\right)\left\{E\left[f^{\prime}\left(W_{y}\right) f^{\prime}\left(W_{y^{\prime}}\right)\right]+E\left[f\left(W_{y}\right) f^{\prime \prime}\left(W_{y^{\prime}}\right)\right]\right\} \\
& +A_{y, y^{\prime}}(4, \varepsilon, j) E\left[f\left(W_{y}\right) f\left(W_{y^{\prime}}\right]\right.
\end{aligned}
$$

with $i, j \in\{1,2\}$ and $i \neq j$. By symmetry we only need to prove the convergence (3.11) with $i=1$ and $j=2$.

Step I. The following convergence holds:

$$
\frac{1}{\varepsilon_{1}^{2} \varepsilon_{2}} \int_{I_{x}} \int_{I_{x}} A_{y, y^{\prime}}\left(4, \varepsilon_{1}, 2\right) E\left[f\left(W_{y}\right) f\left(W_{y^{\prime}}\right)\right] d y d y^{\prime} \longrightarrow 0
$$

as $\varepsilon_{1}, \varepsilon_{2} \rightarrow 0$. We have

$$
\begin{aligned}
A_{y, y^{\prime}} & \left(4, \varepsilon_{1}, 2\right) \\
= & \varepsilon_{2} E\left[\left(W_{y+\varepsilon_{1}}-W_{y}\right)\left(W_{y^{\prime}+\varepsilon_{1}}-W_{y^{\prime}}\right)\right] \\
& -\varepsilon_{1} E\left[\left(W_{y+\varepsilon_{1}}-W_{y}\right)\left(W_{y^{\prime}+\varepsilon_{2}}-W_{y^{\prime}}\right)\right] \\
= & \varepsilon_{2}\left\{E W_{y+\varepsilon_{1}} W_{y^{\prime}+\varepsilon_{1}}-E W_{y} W_{y^{\prime}+\varepsilon_{1}}-E W_{y+\varepsilon_{1}} W_{y^{\prime}}+E W_{y} W_{y^{\prime}}\right\} \\
& -\varepsilon_{1}\left\{E W_{y+\varepsilon_{1}} W_{y^{\prime}+\varepsilon_{2}}-E W_{y} W_{y^{\prime}+\varepsilon_{2}}-E W_{y+\varepsilon_{1}} W_{y^{\prime}}+E W_{y} W_{y^{\prime}}\right\} \\
= & \frac{1}{2 \sqrt{\pi}} \varepsilon_{2}\left(\int_{0}^{t} \frac{2}{\sqrt{r}} e^{-\frac{\left(y-y^{\prime}\right)^{2}}{4 r}} d r-\int_{0}^{t} \frac{1}{\sqrt{r}} e^{-\frac{\left(y-y^{\prime}-\varepsilon_{1}\right)^{2}}{4 r}} d r-\int_{0}^{t} \frac{1}{\sqrt{r}} e^{-\frac{\left(y+\varepsilon_{1}-y^{\prime}\right)^{2}}{4 r}} d r\right) \\
& -\frac{1}{2 \sqrt{\pi}} \varepsilon_{1}\left(\int_{0}^{t} \frac{1}{\sqrt{r}} e^{-\frac{\left(y-y^{\prime}+\varepsilon_{1}-\varepsilon_{2}\right)^{2}}{4 r}} d r-\int_{0}^{t} \frac{1}{\sqrt{r}} e^{-\frac{\left(y-y^{\prime}-\varepsilon_{2}\right)^{2}}{4 r}} d r\right. \\
& \left.-\int_{0}^{t} \frac{1}{\sqrt{r}} e^{-\frac{\left(y+\varepsilon_{1}-y^{\prime}\right)^{2}}{4 r}} d r+\int_{0}^{t} \frac{1}{\sqrt{r}} e^{-\frac{\left(y-y^{\prime}\right)^{2}}{4 r}} d r\right) .
\end{aligned}
$$

Consider the next function on $\mathbb{R}_{+}$(see Sect. 2):

$$
f(x)=x \int_{x}^{\infty} \frac{1}{s^{2}} e^{-\frac{s^{2}}{2}} d s=e^{-\frac{x^{2}}{2}}-x \int_{x}^{\infty} e^{-\frac{s^{2}}{2}} d s .
$$

Then we have

$$
\begin{aligned}
A_{y, y^{\prime}}\left(4, \varepsilon_{1}, 2\right)= & \frac{\sqrt{t}}{\sqrt{2 \pi}} \varepsilon_{2}\left(2 f\left(\frac{y-y^{\prime}}{\sqrt{2 t}}\right)-f\left(\frac{y-y^{\prime}-\varepsilon_{1}}{\sqrt{2 t}}\right)-f\left(\frac{y+\varepsilon_{1}-y^{\prime}}{\sqrt{2 t}}\right)\right) \\
& -\frac{\sqrt{t}}{\sqrt{2 \pi}} \varepsilon_{1}\left(f\left(\frac{y-y^{\prime}+\varepsilon_{1}-\varepsilon_{2}}{\sqrt{2 t}}\right)-f\left(\frac{y-y^{\prime}-\varepsilon_{2}}{\sqrt{2 t}}\right)\right. \\
& \left.-f\left(\frac{y+\varepsilon_{1}-y^{\prime}}{\sqrt{2 t}}\right)+f\left(\frac{y-y^{\prime}}{\sqrt{2 t}}\right)\right) .
\end{aligned}
$$

Notice that, by Taylor's expansion,

$$
f(x)=1-\sqrt{\frac{\pi}{2}} x+\frac{1}{2} x^{2}-\frac{1}{4 !} x^{4}+o\left(x^{4}\right) .
$$


One gets

$$
\begin{aligned}
2 f( & \left.\frac{y-y^{\prime}}{\sqrt{2 t}}\right)-f\left(\frac{y-y^{\prime}-\varepsilon_{1}}{\sqrt{2 t}}\right)-f\left(\frac{y-y^{\prime}+\varepsilon_{1}}{\sqrt{2 t}}\right) \\
= & \frac{1}{4 t}\left\{2\left(y-y^{\prime}\right)^{2}-\left(y-y^{\prime}-\varepsilon_{1}\right)^{2}-\left(y-y^{\prime}+\varepsilon_{1}\right)^{2}\right\} \\
& -\frac{1}{4 \times 4 ! t^{2}}\left\{2\left(y-y^{\prime}\right)^{4}-\left(y-y^{\prime}-\varepsilon_{1}\right)^{4}-\left(y-y^{\prime}+\varepsilon_{1}\right)^{4}\right\}+\alpha_{1} \\
= & -\frac{1}{4 t} \varepsilon_{1}^{2}+\frac{1}{4 \times 4 ! t^{2}}\left(12\left(y-y^{\prime}\right)^{2} \varepsilon_{1}^{2}+2 \varepsilon_{1}^{4}\right)+\alpha_{1}
\end{aligned}
$$

with $\alpha_{1}=\frac{1}{4 t^{2}} o\left(12\left(y-y^{\prime}\right)^{2} \varepsilon_{1}^{2}+2 \varepsilon_{1}^{4}\right)$ and

$$
\begin{aligned}
f( & \left.\frac{y-y^{\prime}+\varepsilon_{1}-\varepsilon_{2}}{\sqrt{2 t}}\right)-f\left(\frac{y-y^{\prime}-\varepsilon_{2}}{\sqrt{2 t}}\right)-f\left(\frac{y-y^{\prime}+\varepsilon_{1}}{\sqrt{2 t}}\right)+f\left(\frac{y-y^{\prime}}{\sqrt{2 t}}\right) \\
= & \frac{1}{4 t}\left\{\left(y-y^{\prime}+\varepsilon_{1}-\varepsilon_{2}\right)^{2}-\left(y-y^{\prime}-\varepsilon_{2}\right)^{2}-\left(y-y^{\prime}+\varepsilon_{1}\right)^{2}+\left(y-y^{\prime}\right)^{2}\right\} \\
& -\frac{1}{4 \times 4 ! t^{2}}\left\{\left(y-y^{\prime}+\varepsilon_{1}-\varepsilon_{2}\right)^{4}-\left(y-y^{\prime}-\varepsilon_{2}\right)^{4}-\left(y-y^{\prime}+\varepsilon_{1}\right)^{4}+\left(y-y^{\prime}\right)^{4}\right\}+\alpha_{2} \\
= & -\frac{1}{4 t} \varepsilon_{1} \varepsilon_{2}+\frac{1}{4 \times 4 ! t^{2}}\left\{12\left(y-y^{\prime}\right)^{2} \varepsilon_{1} \varepsilon_{2}+\varepsilon_{1} \varepsilon_{2}\left(\varepsilon_{1}-\varepsilon_{2}\right)\left(12\left(y-y^{\prime}\right)+2 \varepsilon_{1}-\varepsilon_{2}\right)\right\}+\alpha_{2}
\end{aligned}
$$

with $\alpha_{2}=\frac{1}{4 t^{2}} o\left(12\left(y-y^{\prime}\right)^{2} \varepsilon_{1} \varepsilon_{2}+\varepsilon_{1} \varepsilon_{2}\left(\varepsilon_{1}-\varepsilon_{2}\right)\left(12\left(y-y^{\prime}\right)+2 \varepsilon_{1}-\varepsilon_{2}\right)\right)$. It follows that

$$
\frac{1}{\varepsilon_{1}^{2} \varepsilon_{2}}\left|A_{y, y^{\prime}}\left(4, \varepsilon_{1}, 2\right)\right| \leq \frac{C}{t^{3 / 2}}\left(\varepsilon_{1}+\frac{o\left(\varepsilon_{1} \varepsilon_{2}\right)}{\varepsilon_{1} \varepsilon_{2}}+\frac{o\left(\varepsilon_{1}^{2}\right)}{\varepsilon_{1}^{2}}\right)\left(|x|^{2}+|x|+1\right) .
$$

for all $0<\varepsilon_{2}<\varepsilon_{1}<1$ and $y, y^{\prime} \in I_{x}$, which shows that the convergence (3.11) holds since $f \in \mathscr{H}_{t}$.

Step II. The following convergence holds:

$$
\begin{aligned}
& \frac{1}{\varepsilon_{1}^{2} \varepsilon_{2}} \int_{I_{x}} \int_{I_{x}}\left\{A_{y, y^{\prime}}\left(1, \varepsilon_{1}, 2\right)-A_{y, y^{\prime}}\left(2-1, \varepsilon_{1}, 2\right)\right\} \\
& \quad \times E\left[f^{\prime \prime}\left(W_{y}\right) f\left(W_{y^{\prime}}\right)\right] d y d y^{\prime} \longrightarrow 0,
\end{aligned}
$$

as $\varepsilon_{1}, \varepsilon_{2} \rightarrow 0$. Keeping the notations in Step I, we have

$$
\begin{aligned}
f( & \left.\frac{y-y^{\prime}-\varepsilon}{\sqrt{2 t}}\right)-f\left(\frac{y-y^{\prime}}{\sqrt{2 t}}\right) \\
= & \frac{1}{\sqrt{\pi t}} \varepsilon-\frac{1}{4 t}\left(2\left(y-y^{\prime}\right)-\varepsilon\right) \varepsilon \\
& \quad-\frac{1}{4 \times 4 ! t^{2}}\left(-4\left(y-y^{\prime}\right)^{3}+6 \varepsilon\left(y-y^{\prime}\right)^{2}-4 \varepsilon^{3}\left(y-y^{\prime}\right)+\varepsilon^{3}\right) \varepsilon
\end{aligned}
$$

for all $\varepsilon$ and

$$
\Delta_{1}:=\varepsilon_{2}\left(f\left(\frac{y-y^{\prime}-\varepsilon_{1}}{\sqrt{2 t}}\right)-f\left(\frac{y-y^{\prime}}{\sqrt{2 t}}\right)\right)-\varepsilon_{1}\left(f\left(\frac{y-y^{\prime}-\varepsilon_{2}}{\sqrt{2 t}}\right)-f\left(\frac{y-y^{\prime}}{\sqrt{2 t}}\right)\right)
$$




$$
=\varepsilon_{1} \varepsilon_{2}\left(\varepsilon_{1}-\varepsilon_{2}\right)\left\{\frac{1}{4 t}-\frac{3\left(y-y^{\prime}\right)^{2}}{2 \times 4 ! t^{2}}+\frac{y-y^{\prime}}{\times 4 ! t^{2}}\left(\varepsilon_{1}+\varepsilon_{2}\right)-\frac{1}{4 \times 4 ! t^{2}}\left(\varepsilon_{1}^{2}-\varepsilon_{1} \varepsilon_{2}+\varepsilon_{2}^{2}\right)\right\} \text {. }
$$

It follows from (2.8) that

$$
\begin{aligned}
\mid A_{y, y^{\prime}} & \left(1, \varepsilon_{1}, 2\right)-A_{y, y^{\prime}}\left(2-1, \varepsilon_{1}, 2\right) \mid \\
= & \mid \varepsilon_{2} E\left[W_{y}\left(W_{y^{\prime}+\varepsilon_{1}}-W_{y^{\prime}}\right)\right] E\left[\left(W_{y}-W_{y^{\prime}}\right)\left(W_{y+\varepsilon_{1}}-W_{y}\right)\right] \\
& -\varepsilon_{1} E\left[W_{y}\left(W_{y^{\prime}+\varepsilon_{2}}-W_{y^{\prime}}\right)\right] E\left[\left(W_{y}-W_{y^{\prime}}\right)\left(W_{y+\varepsilon_{1}}-W_{y}\right)\right] \mid \\
= & \left|E\left[\left(W_{y}-W_{y^{\prime}}\right)\left(W_{y+\varepsilon_{1}}-W_{y}\right)\right]\right| \\
& \times\left|\varepsilon_{2} E\left[W_{y}\left(W_{y^{\prime}+\varepsilon_{1}}-W_{y^{\prime}}\right)\right]-\varepsilon_{1} E\left[W_{y}\left(W_{y^{\prime}+\varepsilon_{2}}-W_{y^{\prime}}\right)\right]\right| \\
= & \left|E\left[\left(W_{y}-u_{y^{\prime}}\right)\left(W_{y+\varepsilon_{1}}-W_{y}\right)\right]\right|\left|\Delta_{1}\right| \\
\leq & C\left|y-y^{\prime}\right| \varepsilon_{1}^{2} \varepsilon_{2}\left(\varepsilon_{1}-\varepsilon_{2}\right)\left\{\frac{1}{t}+\frac{1}{t^{2}}\left(|x|^{2}+|x|+1\right)\right\}
\end{aligned}
$$

for all $0<\varepsilon_{2}<\varepsilon_{1}<1$ and $y, y^{\prime} \in I_{x}$, which implies that

$$
\begin{aligned}
& \frac{1}{\varepsilon_{1}^{2} \varepsilon_{2}} \int_{I_{x}} \int_{I_{x}}\left|A_{y, y^{\prime}}\left(1, \varepsilon_{1}, 2\right)-A_{y, y^{\prime}}\left(2-1, \varepsilon_{1}, 2\right)\right|\left|E\left[f^{\prime \prime}\left(W_{y}\right) f\left(W_{y^{\prime}}\right)\right]\right| d y d y^{\prime} \\
& \leq C\left(\varepsilon_{1}-\varepsilon_{2}\right)\left\{\frac{1}{t}+\frac{1}{t^{2}}\left(|x|^{2}+|x|+1\right)\right\} \int_{I_{x}} \int_{I_{x}}\left|y-y^{\prime}\right|\left|E\left[f^{\prime \prime}\left(W_{y}\right) f\left(W_{y^{\prime}}\right)\right]\right| d y d y^{\prime} \\
& \quad \leq C\left(\varepsilon_{1}-\varepsilon_{2}\right)\left\{\frac{1}{t}+\frac{1}{t^{2}}\left(|x|^{2}+|x|+1\right)\right\}\|f\|_{\mathscr{H}_{t}}^{2} \rightarrow 0,
\end{aligned}
$$

as $\varepsilon_{1}, \varepsilon_{2} \rightarrow 0$ since $f \in \mathscr{H}_{t}$. Similarly, we can show the next convergence:

$$
\frac{1}{\varepsilon_{1}^{2} \varepsilon_{2}} \int_{I_{x}} \int_{I_{x}}\left\{A_{y, y^{\prime}}\left(3, \varepsilon_{1}, 2\right)-A_{y, y^{\prime}}\left(2-2, \varepsilon_{1}, 2\right)\right\} E\left[f\left(W_{y}\right) f^{\prime \prime}\left(W_{y^{\prime}}\right)\right] d y d y^{\prime} \longrightarrow 0
$$

as $\varepsilon_{1}, \varepsilon_{2} \rightarrow 0$.

Step III. The following convergence holds:

$$
\begin{aligned}
& \frac{1}{\varepsilon_{1}^{2} \varepsilon_{2}} \int_{I_{x}} \int_{I_{x}} A_{y, y^{\prime}}\left(2-1, \varepsilon_{1}, 2\right) \\
& \quad \times\left\{E\left[f^{\prime}\left(W_{y}\right) f^{\prime}\left(W_{y^{\prime}}\right)\right]+E\left[f^{\prime \prime}\left(W_{y}\right) f\left(W_{y^{\prime}}\right)\right]\right\} d y d y^{\prime} \rightarrow 0
\end{aligned}
$$

as $\varepsilon_{1}, \varepsilon_{2} \rightarrow 0$. By Step II and Lemma 2.3, we have

$$
\begin{aligned}
\left|A_{y, y^{\prime}}\left(2-1, \varepsilon_{1}, 2\right)\right|= & \left|E\left[W_{y^{\prime}}\left(W_{y+\varepsilon_{1}}-W_{y}\right)\right]\right| \\
& \times\left|\varepsilon_{2} E\left[W_{y}\left(W_{y^{\prime}+\varepsilon_{1}}-W_{y^{\prime}}\right)\right]-\varepsilon_{1} E\left[W_{y}\left(W_{y^{\prime}+\varepsilon_{2}}-W_{y^{\prime}}\right)\right]\right| \\
= & \left|E\left[W_{y^{\prime}}\left(W_{y+\varepsilon_{1}}-W_{y}\right)\right]\right|\left|\Delta_{1}\right| \\
\leq & C \varepsilon_{1}^{2} \varepsilon_{2}\left(\varepsilon_{1}-\varepsilon_{2}\right)\left\{\frac{1}{t}+\frac{1}{t^{2}}\left(|x|^{2}+|x|+1\right)\right\}
\end{aligned}
$$

for all $0<\varepsilon_{2}<\varepsilon_{1}<1$ and $y, y^{\prime} \in I_{x}$, which implies that

$$
\frac{1}{\varepsilon_{1}^{2} \varepsilon_{2}} \int_{I_{x}} \int_{I_{x}}\left|A_{y, y^{\prime}}\left(2-1, \varepsilon_{1}, 2\right)\right|\left|E\left[f^{\prime}\left(W_{y}\right) f^{\prime}\left(W_{y^{\prime}}\right)\right]+E\left[f^{\prime \prime}\left(W_{y}\right) f\left(W_{y^{\prime}}\right)\right]\right| d y d y^{\prime}
$$




$$
\leq C\left(\varepsilon_{1}-\varepsilon_{2}\right)\left\{\frac{1}{t}+\frac{1}{t^{2}}\left(|x|^{2}+|x|+1\right)\right\}\|f\|_{\mathscr{H}_{t}}^{2} \longrightarrow 0
$$

as $\varepsilon_{1}, \varepsilon_{2} \rightarrow 0$ by (3.9), since $f \in \mathscr{H}_{t}$. Similarly, we can show that the next convergence:

$$
\begin{aligned}
& \frac{1}{\varepsilon_{1}^{2} \varepsilon_{2}} \int_{I_{x}} \int_{I_{x}} A_{y, y^{\prime}}\left(2-2, \varepsilon_{1}, 2\right) \\
& \quad \times\left\{E\left[f^{\prime}\left(W_{y}\right) f^{\prime}\left(W_{y^{\prime}}\right)\right]+E\left[f\left(W_{y}\right) f^{\prime \prime}\left(W_{y^{\prime}}\right)\right]\right\} d y d y^{\prime} \longrightarrow 0
\end{aligned}
$$

as $\varepsilon_{1}, \varepsilon_{2} \rightarrow 0$. Thus, we have proved the second statement.

Corollary 3.1 Let $f, f_{1}, f_{2}, \ldots \in \mathscr{H}_{t}$ such that $f_{n} \rightarrow f$ in $\mathscr{H}_{t}$. Then the convergence

$$
\left[f_{n}(W), W\right]_{x}^{(S Q)} \longrightarrow[f(W), W]_{x}^{(S Q)}
$$

holds in $L^{2}(\Omega)$ for all $x \in \mathbb{R}$.

\section{The Itô formula for the spatial process}

In this section, as an application of the previous section we discuss the Itô calculus for the process $W=\left\{W_{x},|x| \leq M\right\}$ and fix a time parameter $t>0$. For a continuous process $X$ admitting a finite quadratic variation $[X, X]$, Russo and Vallois [29] have introduced the following Itô formula:

$$
F\left(X_{t}\right)=F\left(X_{0}\right)+\int_{0}^{t} F^{\prime}\left(X_{s}\right) d^{-} X_{s}+\frac{1}{2} \int_{0}^{t} F^{\prime \prime}\left(X_{s}\right) d[X, X]_{s}
$$

for all $F \in C^{2}(\mathbb{R})$, where

$$
\int_{0}^{t} F^{\prime}\left(X_{s}\right) d^{-} X_{s}:=\operatorname{ucp} \lim _{\varepsilon \downarrow 0} \frac{1}{\varepsilon} \int_{0}^{t} F^{\prime}\left(X_{s}\right)\left(X_{s+\varepsilon}-X_{s}\right) d s
$$

is called the forward integral, where the notation ucp lim denotes the uniform convergence in probability on each compact interval. We refer to Russo and Vallois [29] and the references therein for more details of stochastic calculus of continuous processes with finite quadratic variations. It follows from the previous section (the quadratic variation of $\left\{W_{x}, x \in \mathbb{R}\right\}$ is equal to $|x|$ for all $\left.x \in \mathbb{R}\right)$ that

$$
F\left(W_{x}\right)=F\left(W_{0}\right)+\int_{I_{x}} F^{\prime}\left(W_{y}\right) d^{-} W_{y}+\frac{1}{2} \int_{I_{x}} F^{\prime \prime}\left(W_{y}\right) d y
$$

for all $F \in C^{2}(\mathbb{R})$. Thus, by smooth approximating, we have the next Itô type formula.

Theorem 4.1 Let $f \in \mathscr{H}_{t}$ be left continuous. If $F$ is an absolutely continuous function with the derivative $F^{\prime}=f$, then the following Itô type formula holds:

$$
F\left(W_{x}\right)=F\left(W_{0}\right)+\int_{I_{x}} f\left(W_{y}\right) d^{-} W_{y}+\frac{1}{2}[f(W), W]_{x}^{(S Q)} .
$$


Clearly, this is an analogue of Föllmer-Protter-Shiryayev's formula. It is an improvement in terms of the hypothesis on $f$ and it is also quite interesting itself. Some details and more work could be found in Eisenbaum [6], Feng-Zhao [8], Föllmer et al. [9], MoretNualart [18], Peskir [24], Rogers-Walsh [26], Russo-Vallois [28, 29], Yan et al. [36, 37], and the references therein. It is well known that when $W$ is a semimartingale, the forward integral coincides with the Itô integral. However, the following theorem points out that the two integrals are coincident for the process $W=\left\{W_{x}, x \in \mathbb{R}\right\}$. But $W=\left\{W_{x}, x \in \mathbb{R}\right\}$ is not a semimartingale.

Proof of Theorem 4.1 If $f \in C^{1}(\mathbb{R})$, then this is Itô's formula since

$$
[f(W), W]_{x}^{(S Q)}=\int_{I_{x}} f^{\prime}\left(W_{y}\right) d y .
$$

For $f \notin C^{1}(\mathbb{R})$, by a localization argument we may assume that the function $f$ is uniformly bounded. In fact, for any $k \geq 0$ we may consider the set

$$
\Omega_{k}=\left\{\sup _{x \in \mathbb{R}}\left|W_{x}\right|<k\right\}
$$

and let $f^{[k]}$ be a measurable function such that $f^{[k]}=f$ on $[-k, k]$ and such that $f^{[k]}$ vanishes outside. Then $f^{[k]}$ is uniformly bounded and $f^{[k]} \in \mathscr{H}_{t}$ for every $k \geq 0$. Set $\frac{d}{d x} F^{[k]}=f^{[k]}$ and $F^{[k]}=F$ on $[-k, k]$. If the theorem is true for all uniformly bounded functions on $\mathscr{H}_{t}$, then we get the desired formula

$$
F^{[k]}\left(W_{x}\right)=F^{[k]}\left(W_{0}\right)+\int_{I_{x}} f^{[k]}\left(W_{y}\right) d^{-} W_{y}+\frac{1}{2}\left[f^{[k]}(W), W\right]_{x}^{(S Q)}
$$

on the set $\Omega_{k}$. Letting $k$ tend to infinity we deduce the Itô formula (4.1).

Let now $F^{\prime}=f \in \mathscr{H}_{t}$ be uniformly bounded and left continuous. Consider the function $\zeta$ on $\mathbb{R}$ by

$$
\zeta(x):= \begin{cases}c e^{\frac{1}{(x-1)^{2}-1}} & x \in(0,2) \\ 0 & \text { otherwise }\end{cases}
$$

where $c$ is a normalizing constant such that $\int_{\mathbb{R}} \zeta(x) d x=1$. Define the mollifiers

$$
\zeta_{n}(x):=n \zeta(n x), \quad n=1,2, \ldots
$$

and the sequence of smooth functions

$$
F_{n}(x):=\int_{\mathbb{R}} F(x-y) \zeta_{n}(y) d y, \quad x \in \mathbb{R} .
$$

Then $F_{n} \in C^{\infty}(\mathbb{R})$ for all $n \geq 1$ and the Itô formula

$$
F_{n}\left(W_{x}\right)=F_{n}\left(W_{0}\right)+\int_{I_{x}} f_{n}\left(W_{y}\right) d^{-} W_{y}+\frac{1}{2} \int_{0}^{t} f_{n}^{\prime}\left(W_{y}\right) d y
$$


holds for all $n \geq 1$, where $f_{n}=F_{n}^{\prime}$. Moreover, by using Lebesgue's dominated convergence theorem, one can prove that, as $n \rightarrow \infty$, for each $x$,

$$
F_{n}(x) \longrightarrow F(x), \quad f_{n}(x) \longrightarrow f(x),
$$

and $\left\{f_{n}\right\} \subset \mathscr{H}_{t}, f_{n} \rightarrow f$ in $\mathscr{H}_{t}$. It follows that

$$
\frac{1}{2} \int_{0}^{t} f_{n}^{\prime}\left(W_{y}\right) d y=\left[f_{n}(W), W\right]_{x}^{(S Q)} \longrightarrow[f(W), W]_{x}^{(S Q)}
$$

and

$$
f_{n}\left(W_{x}\right) \longrightarrow f\left(W_{x}\right)
$$

in $L^{2}(\Omega)$ by Corollary 3.1, as $n$ tends to infinity. It follows that

$$
\begin{aligned}
\int_{I_{x}} f_{n}\left(W_{y}\right) d^{-} W_{y} & =F_{n}\left(W_{y}\right)-F_{n}\left(W_{0}\right)-\frac{1}{2}\left[f_{n}(W), W\right]_{x}^{(S Q)} \\
& \longrightarrow F\left(W_{y}\right)-F_{n}\left(W_{0}\right)-\frac{1}{2}[f(W), W]_{x}^{(S Q)}
\end{aligned}
$$

in $L^{2}(\Omega)$, as $n$ tends to infinity. This completes the proof since the integral is closed in $L^{2}(\Omega)$.

Now, we consider the Itô formula including the Skorohod integral of the spatial process $W=\left\{W_{x}\right\}$.

Theorem 4.2 Let $f$ be left continuous. If $F$ is an absolutely continuous function with the derivative $F^{\prime}=f$ satisfying the condition

$$
|F(y)|,|f(y)| \leq C e^{\beta y^{2}}, \quad y \in \mathbb{R},
$$

with $0 \leq \beta<\frac{\sqrt{\pi}}{4 \sqrt{t}}$, then the following Itô type formula holds:

$$
F\left(W_{x}\right)=F\left(W_{0}\right)+\int_{I_{x}} f\left(W_{y}\right) \delta W_{y}+\frac{1}{2}[f(W .), W]_{x}^{(S Q)}
$$

for all $x \in[-M, M]$.

According to the two theorems above we get the next relationship:

$$
\int_{I_{x}} f\left(W_{y}\right) \delta W_{y}=\int_{I_{x}} f\left(W_{y}\right) d^{-} W_{y}
$$

if $f$ satisfies the growth condition (4.6). Similar to the proof of Theorem 4.1 one can introduce Theorem 4.2. But we need to give the following standard Itô type formula:

$$
F\left(W_{x}\right)=F\left(W_{0}\right)+\int_{I_{x}} F^{\prime}\left(W_{y}\right) \delta W_{y}+\frac{1}{2} \int_{I_{x}} F^{\prime \prime}\left(W_{y}\right) d y
$$


for all $F \in C^{2}(\mathbb{R})$ satisfying the condition

$$
|F(y)|,\left|F^{\prime}(y)\right|,\left|F^{\prime \prime}(y)\right| \leq C e^{\beta y^{2}}, \quad y \in \mathbb{R}
$$

with $0 \leq \beta<\frac{\sqrt{\pi}}{4 \sqrt{t}}$. It is important to note that one have given a standard Itô formula for a large class of Gaussian processes in Alós et al. [1]. However, the process $x \mapsto W_{x}$ does not satisfy the condition in Alós et al. [1] since

$$
E\left[u(t, x)^{2}\right]=\sqrt{\frac{t}{\pi}}, \quad \frac{d}{d x} E\left[u(t, x)^{2}\right]=0
$$

for all $t \geq 0$ and $x \in \mathbb{R}$. So, we need to give the proof of the formula (4.9).

Proof of (4.9) Let us fix $x \in[-M, M]$ and let $\pi \equiv\left\{x_{j}^{n}=\frac{j x}{n} ; j=0,1, \ldots, n\right\}$ be a partition of $[0, x]$. Clearly, the growth condition (4.6) implies that

$$
E\left[\sup _{x \in \mathbb{R}}\left|G\left(W_{x}\right)\right|^{p}\right] \leq c^{p} E\left[e^{p \beta \sup _{x \in \mathbb{R}}\left|W_{x}\right|}\right]<\infty
$$

for some constant $c>0$ and all $p<\frac{\sqrt{\pi}}{2 \beta \sqrt{t}}$, where $G \in\left\{F, F^{\prime}, F^{\prime \prime}\right\}$. In particular, the estimate (4.11) holds for $p=2$. Using the Taylor expansion, we have

$$
\begin{aligned}
F\left(W_{x}\right)= & F\left(W_{0}\right)+\sum_{j=1}^{n} F^{\prime}\left(W_{x_{j-1}^{n}}\right)\left(W_{x_{j}^{n}}-W_{x_{j-1}^{n}}\right) \\
& +\frac{1}{2} \sum_{j=1}^{n} F^{\prime \prime}\left(W_{j}\left(\theta_{j}\right)\right)\left(W_{x_{j}^{n}}-W_{x_{j-1}^{n}}\right)^{2} \\
\equiv & F\left(W_{0}\right)+I^{n}+J^{n},
\end{aligned}
$$

where $W_{j}\left(\theta_{j}\right)=W_{x_{j-1}^{n}}+\theta_{j}\left(W_{x_{j}^{n}}-W_{x_{j-1}^{n}}\right)$ with $\theta_{j} \in(0,1)$ being a random variable. By the duality relationship (2.1) we have

$$
\begin{aligned}
I^{n}= & \sum_{j=1}^{n} F^{\prime}\left(W_{x_{j-1}^{n}}\right)\left(\delta^{t}\left(1_{\left(x_{j-1}^{n}, x_{j}^{n}\right]}\right)\right) \\
= & \delta^{t}\left(\sum_{j=1}^{n} f^{\prime}\left(W_{x_{j-1}^{n}}\right) 1_{\left(x_{j-1}^{n}, x_{j}\right]}(\cdot)\right) \\
& +\sum_{j=1}^{n} F^{\prime \prime}\left(W_{\left.x_{j-1}^{n}\right)}\left\langle 1_{\left(0, x_{j-1}^{n}\right]}, 1_{\left(x_{j-1}^{n}, x_{j}^{n}\right]}\right\rangle_{\mathcal{H}}\right. \\
\equiv & I_{1}^{n}+I_{2}^{n} .
\end{aligned}
$$

Now, in order to end the proof we claim that the following convergences in $L^{2}$ hold:

$$
\begin{aligned}
& I_{2}^{n} \longrightarrow-\frac{1}{2} \int_{0}^{t} F^{\prime \prime}\left(W_{y}\right) d y, \\
& I_{1}^{n} \longrightarrow \int_{I_{x}} F^{\prime}\left(W_{y}\right) \delta W_{y},
\end{aligned}
$$




$$
J^{n} \longrightarrow \int_{0}^{t} F^{\prime \prime}\left(W_{y}\right) d y
$$

as $n$ tends to infinity.

To prove the first convergence, it is enough to establish that

$$
\Lambda_{n}:=E\left|I_{2}^{n}+\frac{1}{2} \sum_{j=1}^{n} F^{\prime \prime}\left(W_{x_{j}^{n}}\right)\left(x_{j}^{n}-x_{j-1}^{n}\right)\right|^{2} \longrightarrow 0
$$

as $n$ tends to infinity. By the -Minkowski inequality we have

$$
\begin{aligned}
& \sqrt{\Lambda_{n}}=\left(E\left|\sum_{j=1}^{n} F^{\prime \prime}\left(W_{x_{j-1}^{n}}\right)\left\{\left\langle 1_{\left(0, x_{j-1}^{n}\right]}, 1_{\left(x_{j-1}^{n}, x_{j}^{n}\right]}\right\rangle_{\mathcal{H}_{\Delta}}+\frac{1}{2}\left(x_{j}^{n}-x_{j-1}^{n}\right)\right\}\right|^{2}\right)^{1 / 2} \\
& \leq C \sum_{j=1}^{n}\left|\left\langle 1_{\left(0, x_{j-1}^{n}\right]}, 1_{\left(x_{j-1}^{n}, x_{j}^{n}\right]}\right\rangle_{\mathcal{H}_{\Delta}}+\frac{1}{2}\left(x_{j}^{n}-x_{j-1}^{n}\right)\right| \\
& =C \sum_{j=1}^{n}\left|\frac{1}{2 \sqrt{\pi}} \int_{0}^{t} \frac{1}{\sqrt{r}}\left(1-e^{-\frac{1}{4 r}\left(x_{j}^{n}-x_{j-1}^{n}\right)^{2}}\right) d r-\frac{1}{2}\left(x_{j}^{n}-x_{j-1}^{n}\right)\right| \\
& =C \sum_{j=1}^{n}\left|x_{j}^{n}-x_{j-1}^{n}\right|\left|\frac{1}{\sqrt{2 \pi}} \int_{\frac{x_{j}^{n}-x_{j-1}^{n}}{\sqrt{2 t}}}^{\infty} \frac{1}{s^{2}}\left(1-e^{-\frac{s^{2}}{2}}\right) d s-\frac{1}{2}\right| \\
& =C \sum_{j=1}^{n}\left|x_{j}^{n}-x_{j-1}^{n}\right|\left|\frac{1}{\sqrt{2 \pi}} \int_{0}^{\frac{x_{j}^{n}-x_{j-1}^{n}}{\sqrt{2 t}}} \frac{1}{s^{2}}\left(1-e^{-\frac{s^{2}}{2}}\right) d s\right| \\
& =C|x| \frac{1}{\sqrt{2 \pi}} \int_{0}^{\frac{|x|}{n \sqrt{2 t}}} \frac{1}{s^{2}}\left(1-e^{-\frac{s^{2}}{2}}\right) d s \rightarrow 0 \quad(n \rightarrow \infty) .
\end{aligned}
$$

Now, we prove the third convergence. We have

$$
\begin{aligned}
\Lambda_{n}(2) & :=E\left|J^{n}-\int_{I_{x}} F^{\prime \prime}\left(W_{y}\right) d y\right| \\
& =E\left|\frac{1}{2} \sum_{j=1}^{n} F^{\prime \prime}\left(W_{j}\left(\theta_{j}\right)\right)\left(W_{x_{j}^{n}}-W_{x_{j-1}^{n}}\right)^{2}-\int_{I_{x}} F^{\prime \prime}\left(W_{y}\right) d y\right| .
\end{aligned}
$$

Suppose that $n \geq m$, and for any $j=1, \ldots, n$ we denote by $x_{j}^{m(n)}$ the point of the $m$ th partition that is closer to $x_{j}^{n}$ from the left hand. Then we obtain

$$
\begin{aligned}
\Lambda_{n}(2) \leq & \frac{1}{2} E\left|\sum_{j=1}^{n}\left(F^{\prime \prime}\left(W_{j}\left(\theta_{j}\right)\right)-F^{\prime \prime}\left(W_{x_{j}^{m(n)}}\right)\right)\left(W_{x_{j}^{n}}-W_{x_{j-1}^{n}}\right)^{2}\right| \\
& +\frac{1}{2} E\left|\sum_{k=1}^{m} F^{\prime \prime}\left(W_{x_{j}^{m(n)}}\right) \sum_{\left\{j: x_{k-1}^{m(n)} \leq x_{j-1}^{m(n)}<x_{k}^{m(n)}\right\}}\left(\left(W_{x_{j}^{n}}-W_{x_{j-1}^{n}}\right)^{2}-\left(x_{j}^{n}-x_{j-1}^{n}\right)\right)\right| \\
& +E\left|\sum_{k=1}^{m} \int_{x_{k-1}^{n}}^{x_{k}^{n}}\left(F^{\prime \prime}\left(W_{x_{j}^{m(n)}}\right)-F^{\prime \prime}\left(W_{y}\right)\right) d y\right|
\end{aligned}
$$




$$
\equiv \frac{1}{2} \Lambda_{n}(2,1)+\frac{1}{2} \Lambda_{n}(2,2)+\Lambda_{n}(2,3)
$$

Clearly, we have $\Lambda_{n}(2,2) \rightarrow 0(n, m \rightarrow \infty)$ by Lemma 3.1 and the estimate (4.11),

$$
\Lambda_{n}(2,3) \leq|x| E \sup _{|z-y| \leq \frac{|x|}{m}}\left|F^{\prime \prime}\left(W_{z}\right)-F^{\prime \prime}\left(W_{y}\right)\right| \longrightarrow 0 \quad(m \rightarrow \infty)
$$

and

$$
\begin{aligned}
& \Lambda_{n}(2,1)=E\left|\sum_{j=1}^{n}\left(F^{\prime \prime}\left(W_{j}\left(\theta_{j}\right)\right)-F^{\prime \prime}\left(W_{x_{j}^{m}}\right)\right)\left(W_{x_{j}}-W_{x_{j-1}}\right)^{2}\right| \\
& \leq C E\left\{\sup _{|z-y| \leq \frac{|x|}{n}}\left|F^{\prime \prime}\left(W_{z}\right)-F^{\prime \prime}\left(W_{y}\right)\right| \sum_{j=1}^{n}\left(W_{x_{j}^{n}}-W_{x_{j-1}^{n}}\right)^{2}\right\} \\
& \leq C\left\{E \sup _{|z-y| \leq \frac{|x|}{n}}\left|F^{\prime \prime}\left(W_{z}\right)-F^{\prime \prime}\left(W_{y}\right)\right|^{2} E\left(\sum_{j=1}^{n}\left(W_{x_{j}^{n}}-W_{x_{j-1}^{n}}\right)^{2}\right)^{2}\right\}^{1 / 2} \\
& \leq C|x|\left\{E \sup _{|z-y| \leq \frac{|x|}{n}}\left|F^{\prime \prime}\left(W_{z}\right)-F^{\prime \prime}\left(W_{y}\right)\right|^{2}\right\}^{1 / 2} \rightarrow 0 \quad(n \rightarrow \infty)
\end{aligned}
$$

by (2.4), the estimate (4.11) and Minkowski's inequality. Thus, we obtain the third convergence, i.e., $J^{n} \rightarrow \int_{0}^{t} F^{\prime \prime}\left(W_{y}\right) d y$ in $L^{1}$.

Finally, to end the proof we address the second convergence:

$$
I_{1}^{n}=\delta^{\Delta}\left(\sum_{j=1}^{n} F^{\prime}\left(W_{x_{j-1}^{n}}\right) 1_{\left(x_{j-1}^{n}, x_{j}^{n}\right]}(\cdot)\right) \rightarrow \int_{I_{x}} F^{\prime}\left(W_{y}\right) \delta W_{y} \quad(n \rightarrow \infty) .
$$

We need to show that

$$
A_{n}:=\sum_{j=1}^{n} F^{\prime}\left(W_{x_{j-1}^{n}}\right) 1_{\left(x_{j-1}^{n}, x_{j}^{n}\right]}(\cdot) \longrightarrow F^{\prime}(W .) 1_{I_{x}}(\cdot)
$$

in $L^{2}\left(\Omega ; \mathcal{H}_{\Delta}\right)$, as $n$ tends to infinity. We have

$$
\begin{aligned}
& E\left\|A_{n}-F^{\prime}(W .) 1_{I_{x}}(\cdot)\right\|_{\mathcal{H}_{\Delta}}^{2} \\
& =E\left\|\sum_{j=1}^{n}\left(F^{\prime}\left(W_{x_{j-1}}\right)-F^{\prime}(W .)\right) 1_{\left(x_{j-1}^{n}, x_{j}^{n}\right]}(\cdot)\right\|_{\mathcal{H}_{\Delta}}^{2} \\
& \quad \leq E\left(\sup _{|u-v| \leq \frac{|x|}{n}}\left|F^{\prime}\left(W_{u}\right)-F^{\prime}\left(W_{v}\right)\right|^{2}\right)\left\|\sum_{j=1}^{n} 1_{\left(x_{j-1}^{n}, x_{j}^{n}\right]}(\cdot)\right\|_{\mathcal{H}_{\Delta}}^{2},
\end{aligned}
$$

for all $n \geq 1$ and $x \in \mathbb{R}$. Notice that

$$
\left\|\sum_{j=1}^{n} 1_{\left(x_{j-1}^{n}, x_{j}^{n}\right]}(\cdot)\right\|_{\mathcal{H}_{\Delta}}^{2}
$$




$$
\begin{aligned}
= & \left\langle\sum_{j=1}^{n} 1_{\left(x_{j-1}^{n}, x_{j}^{n}\right]}(\cdot), \sum_{j=1}^{n} 1_{\left(x_{j-1}^{n}, x_{j}^{n}\right]}(\cdot)\right\rangle_{\mathcal{H}_{\Delta}} \\
= & \sum_{j=1}^{n}\left\langle 1_{\left(x_{j-1}^{n}, x_{j}^{n}\right]}(\cdot),\left.1_{\left(x_{j-1}^{n}, x_{j}^{n}\right]}(\cdot)\right|_{\mathcal{H}_{\Delta}}+2 \sum_{1 \leq i<j \leq n}\left\langle 1_{\left(x_{j-1}^{n}, x_{j}^{n}\right]}(\cdot), 1_{\left(x_{i-1}^{n}, x_{i}^{n}\right]}(\cdot)\right\rangle_{\mathcal{H} \Delta}\right. \\
= & 2 \sum_{j=1}^{n} E\left[\left(u\left(t, x_{j}^{n}\right)-u\left(t, x_{j-1}^{n}\right)\right)^{2}\right] \\
& +2 \sum_{1 \leq i<j \leq n} E\left[\left(u\left(t, x_{j}^{n}\right)-u\left(t, x_{j-1}^{n}\right)\right)\left(u\left(t, x_{i}^{n}\right)-u\left(t, x_{i-1}^{n}\right)\right)\right] \\
\leq & C_{t} \sum_{j=1}^{n}\left|x_{j}^{n}-x_{j-1}^{n}\right|+C_{t} \sum_{1 \leq i<j \leq n}\left|\left(x_{j}^{n}-x_{j-1}^{n}\right)\left(x_{i}^{n}-x_{i-1}^{n}\right)\right| \leq C_{t}|x|
\end{aligned}
$$

by (2.6) and (2.8). Combining this with (4.16) and the estimate (4.11), we get

$$
A_{n}:=\sum_{j=1}^{n} F^{\prime}\left(W_{x_{j-1}^{n}}\right) 1_{\left(x_{j-1}^{n}, x_{j}^{n}\right]}(\cdot) \longrightarrow F^{\prime}(W .) 1_{I_{x}}(\cdot)
$$

in $L^{2}\left(\Omega ; \mathcal{H}_{\Delta}\right)$, as $n$ tends to infinity. It follows that

$$
I_{1}^{n}=F\left(W_{x}\right)-F\left(W_{0}\right)-I_{2}^{n}-J^{n} \longrightarrow F\left(W_{x}\right)-F\left(W_{0}\right)-\frac{1}{2} \int_{I_{x}} F^{\prime \prime}\left(W_{y}\right) d y
$$

in $L^{2}(\Omega)$, as $n$ tends to infinity. This completes the proof since the integral $\int_{0}^{\cdot} u_{s} \delta W_{s}$ is closed in $L^{2}(\Omega)$.

\section{The Bouleau-Yor identity for the spatial process}

In this section, we consider the local time of the process $W=\left\{W_{x}, x \in[0, M]\right\}$. Our main object is to prove that the integral

$$
\int_{\mathbb{R}} g(a) \mathscr{L}^{\Delta}(x, d a)
$$

is well-defined and that the identity

$$
\int_{\mathbb{R}} g(a) \mathscr{L}^{\Delta}(x, d a)=-[g(u(t, \cdot)), u(t, \cdot)]_{x}^{(S Q)}
$$

holds for all $g \in \mathscr{H}_{t}$ and $t>0$, where

$$
\mathscr{L}^{\Delta}(x, a)=\int_{I_{x}} \delta(u(t, y)-a) d y .
$$

is the local time of $W=\left\{W_{x}, x \in[0, M]\right\}$. The identity (5.1) is called the Bouleau-Yor identity. More work on this subject can be found in Bouleau-Yor [3], Eisenbaum [6], Föllmer et al. [9], Feng-Zhao [8], Peskir [24], Rogers-Walsh [26], Yan et al. [36, 37], and the references therein. 
Recall that, for any closed interval $I \subset \mathbb{R}_{+}$and for any $a \in \mathbb{R}$, the local time $L(a, I)$ of $u$ is defined as the density of the occupation measure $\mu_{I}$ defined by

$$
\mu_{I}(A)=\int_{I} 1_{A}\left(W_{x}\right) d x
$$

It can be shown (see Geman and Horowitz [10], Theorem 6.4) that the following occupation density formula holds:

$$
\int_{I} g\left(W_{x}, x\right) d x=\int_{\mathbb{R}} d a \int_{I} g(a, x) L(a, d x)
$$

for every Borel function $g(a, x) \geq 0$ on $I \times \mathbb{R}$. Thus, some estimates in Sect. 2 and Theorem 21.9 in Geman-Horowitz [10] together imply that the following result holds.

Corollary 5.1 The local time $\mathscr{L}^{\Delta}(a, x):=L(a,[0, x])$ of $W=\left\{W_{x},|x| \leq M\right\}$ exists and $\mathscr{L}^{t} \in$ $L^{2}(\lambda \times P)$ for all $x \in[0, M]$ and $(a, x) \mapsto \mathscr{L}^{\Delta}(a, x)$ is jointly continuous, where $\lambda$ denotes Lebesgue measure. Moreover, the occupation formula

$$
\int_{0}^{t} \psi\left(W_{x}, x\right) d x=\int_{\mathbb{R}} d a \int_{0}^{t} \psi(a, x) \mathscr{L}^{\Delta}(a, d x)
$$

holds for every continuous and bounded function $\psi(a, x): \mathbb{R} \times \mathbb{R}_{+} \rightarrow \mathbb{R}$ and any $x \in[0, M]$.

Lemma 5.1 For any $f_{\triangle}=\sum_{j} f_{j} 1_{\left(a_{j-1}, a_{j}\right]} \in \mathscr{E}$, we define

$$
\int_{\mathbb{R}} f_{\Delta}(y) \mathscr{L}^{\Delta}(d y, x):=\sum_{j} f_{j}\left[\mathscr{L}^{t}\left(a_{j}, x\right)-\mathscr{L}^{\Delta}\left(a_{j-1}, x\right)\right]
$$

Then the integral is well-defined and

$$
\int_{\mathbb{R}} f_{\Delta}(y) \mathscr{L}^{\Delta}(d y, x)=-\left[f_{\Delta}(W), W\right]_{x}^{(S Q)}
$$

almost surely, for all $x \in[0, M]$.

Proof For the function $f_{\triangle}(y)=1_{(a, b]}(y)$ we define the sequence of smooth functions $f_{n}, n=$ $1,2, \ldots$, by

$$
f_{n}(y)=\int_{\mathbb{R}} f_{\triangle}(y-z) \zeta_{n}(z) d z=\int_{a}^{b} \zeta_{n}(y-z) d z
$$

for all $y \in \mathbb{R}$, where $\zeta_{n}, n \geq 1$ are the so-called mollifiers given in (4.4). Then $\left\{f_{n}\right\} \subset C^{\infty}(\mathbb{R}) \cap$ $\mathscr{H}_{t}$ and $f_{n}$ converges to $f_{\triangle}$ in $\mathscr{H}_{t}$, as $n$ tends to infinity. It follows from the occupation formula that

$$
\begin{aligned}
{\left[f_{n}(W), W\right]_{x}^{(S Q)} } & =\int_{0}^{x} f_{n}^{\prime}\left(W_{y}\right) d y \\
& =\int_{\mathbb{R}} f_{n}^{\prime}(y) \mathscr{L}^{\Delta}(y, x) d y=\int_{\mathbb{R}}\left(\int_{a}^{b} \zeta_{n}^{\prime}(y-z) d z\right) \mathscr{L}^{\Delta}(y, x) d y
\end{aligned}
$$




$$
\begin{aligned}
& =-\int_{\mathbb{R}} \mathscr{L}^{t}(y, x)\left(\zeta_{n}(y-b)-\zeta_{n}(y-a)\right) d y \\
& =\int_{\mathbb{R}} \mathscr{L}^{\Delta}(y, x) \zeta_{n}(y-a) d y-\int_{\mathbb{R}} \mathscr{L}^{\Delta}(y, x) \zeta_{n}(y-b) d y \\
& \longrightarrow \mathscr{L}^{\Delta}(a, x)-\mathscr{L}^{\Delta}(b, x)
\end{aligned}
$$

almost surely, as $n \rightarrow \infty$, by the continuity of $y \mapsto \mathscr{L}^{\Delta}(y, x)$. On the other hand, we see also that there exists a subsequence $\left\{f_{n_{k}}\right\}$ such that

$$
\left[f_{n_{k}}(W), W\right]_{x}^{(S Q)} \longrightarrow\left[1_{(a, b]}(W), W\right]_{x}^{(S Q)}
$$

for all $x \geq 0$, almost surely, as $k \rightarrow \infty$ since $f_{n}$ converges to $f_{\triangle}$ in $\mathscr{H}_{t}$. It follows that

$$
\left[1_{(a, b]}(W), W\right]_{x}^{(S Q)}=\left(\mathscr{L}^{\Delta}(a, x)-\mathscr{L}^{\Delta}(b, x)\right)
$$

for all $x \geq 0$, almost surely. Thus, the identity

$$
\sum_{j} f_{j}\left[\mathscr{L}^{\Delta}\left(a_{j}, x\right)-\mathscr{L}^{\Delta}\left(a_{j-1}, x\right)\right]=-\left[f_{\Delta}(W), W\right]_{x}^{(S Q)}
$$

follows from the linearity property, and the lemma follows.

As a direct consequence of the above lemma, for every $f \in \mathscr{H}_{t}$ if

$$
\lim _{n \rightarrow \infty} f_{\triangle, n}(y)=\lim _{n \rightarrow \infty} g_{\triangle, n}(x)=f(y)
$$

in $\mathscr{H}_{t}$, where $\left\{f_{\triangle, n}\right\},\left\{g_{\triangle, n}\right\} \subset \mathscr{E}$, we then have

$$
\begin{aligned}
& \lim _{n \rightarrow \infty} \int_{\mathbb{R}} f_{\triangle, n}(y) \mathscr{L}^{\Delta}(y, x) d y \\
& =-\lim _{n \rightarrow \infty}\left[f_{\triangle, n}(W), W\right]_{x}^{(S Q)}=-[f(W), W]_{x}^{(S Q)} \\
& =-\lim _{n \rightarrow \infty}\left[g_{\triangle, n}(W), W\right]_{x}^{(S Q)}=\lim _{n \rightarrow \infty} \int_{\mathbb{R}} g_{\triangle, n}(y) \mathscr{L}^{\Delta}(y, x) d y
\end{aligned}
$$

in $L^{2}(\Omega)$. Thus, by the denseness of $\mathscr{E}$ in $\mathscr{H}_{t}$ we can define

$$
\int_{\mathbb{R}} f(y) \mathscr{L}^{\Delta}(d y, x):=\lim _{n \rightarrow \infty} \int_{\mathbb{R}} f_{\triangle, n}(y) \mathscr{L}^{\Delta}(d y, x)
$$

for any $f \in \mathscr{H}_{t}$, where $\left\{f_{\triangle, n}\right\} \subset \mathscr{E}$ and

$$
\lim _{n \rightarrow \infty} f_{\triangle, n}=f
$$

in $\mathscr{H}_{t}$. The considerations are enough to prove the following theorem.

Theorem 5.1 For any $f \in \mathscr{H}_{t}$, the integral

$$
\int_{\mathbb{R}} f(y) \mathscr{L}^{\Delta}(d y, x)
$$


is well-defined in $L^{2}(\Omega)$ and the Bouleau-Yor type identity

$$
[f(W), W]_{x}^{(S Q)}=-\int_{\mathbb{R}} f(y) \mathscr{L}^{\Delta}(d y, x)
$$

holds, almost surely, for all $x \in[0, M]$.

Corollary 5.2 (Tanaka formula) For any $a \in \mathbb{R}$ we have

$$
\begin{aligned}
& \left(W_{x}-a\right)^{+}=\left(W_{0}-a\right)^{+}+\int_{0}^{x} 1_{\left\{W_{y}>a\right\}} d^{-} W_{y}+\frac{1}{2} \mathscr{L}^{\Delta}(a, x), \\
& \left(W_{x}-a\right)^{-}=\left(W_{0}-a\right)^{-}-\int_{0}^{x} 1_{\left\{W_{y}<a\right\}} d^{-} W_{y}+\frac{1}{2} \mathscr{L}^{\Delta}(a, x), \\
& \left|W_{x}-a\right|=\left|W_{0}-a\right|+\int_{0}^{x} \operatorname{sign}\left(W_{x}-a\right) d^{-} W_{y}+\mathscr{L}^{\Delta}(a, x) .
\end{aligned}
$$

Proof Take $F(y)=(y-x)^{+}$. Then $F$ is absolutely continuous and

$$
F(x)=\int_{-\infty}^{y} 1_{(x, \infty)}(y) d y .
$$

It follows from the identity (5.3) and Itô's formula (4.2) that

$$
\begin{aligned}
\mathscr{L}^{\Delta}(a, x) & =\left[1_{(a,+\infty)}(W), W\right]_{x}^{(S Q)} \\
& =2\left(W_{x}-a\right)^{+}-2(-a)^{+}-2 \int_{0}^{x} 1_{\left\{W_{y}>a\right\}} d^{-} W_{y}
\end{aligned}
$$

for all $x \geq 0$, which gives the first identity. In the same way one can obtain the second identity, and by subtracting the last identity from the previous one, we get the third identity.

According to Theorem 5.1, we get an analogue of the Itô formula (Bouleau-Yor type formula).

Corollary 5.3 Let $f \in \mathscr{H}_{t}$ be a left continuous function with right limits. If $F$ is an absolutely continuous function with $F^{\prime}=f$, then the Itô type formula

$$
F\left(W_{x}\right)=F\left(W_{0}\right)+\int_{0}^{x} f\left(W_{y}\right) d^{-} W_{y}-\frac{1}{2} \int_{\mathbb{R}} f(y) \mathscr{L}^{\Delta}(d y, x)
$$

holds for all $x \geq 0$.

Recall that, if $F$ is the difference of two convex functions, then $F$ is an absolutely continuous function with derivative of bounded variation. Thus, the Itô-Tanaka formula

$$
\begin{aligned}
F\left(W_{x}\right) & =F(0)+\int_{0}^{x} F^{\prime}\left(W_{y}\right) d^{-} W_{y}+\frac{1}{2} \int_{\mathbb{R}} \mathscr{L}^{\Delta}(y, x) F^{\prime \prime}(d y) \\
& \equiv F(0)+\int_{0}^{x} F^{\prime}\left(W_{y}\right) d^{-} W_{y}-\frac{1}{2} \int_{\mathbb{R}} F^{\prime}(y) \mathscr{L}^{\Delta}(d y, x)
\end{aligned}
$$

holds. 


\section{The quadratic covariation of process $B=\left\{B_{t}, t \geq 0\right\}$}

In this section, we study the existence of the PQC $[f(B), B]^{(T Q)}$. Recall that

$$
I_{\varepsilon}^{2}(f, x, t)=\frac{1}{\sqrt{\varepsilon}} \int_{0}^{t}\{f(u(s+\varepsilon, x))-f(u(s, x))\}(u(s+\varepsilon, x)-u(s, x)) \frac{d s}{2 \sqrt{s}}
$$

for $\varepsilon>0, t \geq 0$ and $x \in \mathbb{R}$, and

$$
[f(u(\cdot, x)), u(\cdot, x)]_{t}^{(T Q)}=\lim _{\varepsilon \downarrow 0} I_{\varepsilon}^{2}(f, x, t)
$$

provided the limit exists in probability. In this section, we study some analysis questions of the process $\{u(t, \cdot), t \geq 0\}$ associated with the quadratic covariation $[f(u(\cdot, x)), u(\cdot, x)]^{(T Q)}$, and the research includes the existence of the PQC $[f(u(\cdot, x)), u(\cdot, x)]^{(T Q)}$, the Itô and Tanaka formulas. Recall that $B_{t}=u(t, \cdot)(t \in[0, T])$ and

$$
E\left[B_{t}^{2}\right]=\sqrt{\frac{t}{\pi}}
$$

for all $t \geq 0$ and $x \in \mathbb{R}$. It follows from Alós et al. [1] that the Itô formula holds,

$$
f\left(B_{t}\right)=f(0)+\int_{0}^{t} f^{\prime}\left(B_{s}\right) \delta B_{s}+\frac{1}{2 \sqrt{2}} \int_{0}^{t} f^{\prime \prime}\left(B_{s}\right) \frac{d s}{\sqrt{2 \pi s}}
$$

for all $t \in[0, T]$ and $f \in C^{2}(\mathbb{R})$ satisfying the condition

$$
|f(x)|,\left|f^{\prime}(x)\right|,\left|f^{\prime \prime}(x)\right| \leq C e^{\beta x^{2}}, \quad x \in \mathbb{R}
$$

with $0 \leq \beta<\frac{\sqrt{\pi}}{4 \sqrt{T}}$.

Recall that the local Hölder index $\gamma_{0}$ of a continuous paths process $\left\{X_{t}: t \geq 0\right\}$ is the supremum of the exponents $\gamma$ verifying, for any $T>0$,

$$
P\left(\left\{\omega: \exists L(\omega)>0, \forall s, t \in[0, T],\left|X_{t}(\omega)-X_{s}(\omega)\right| \leq L(\omega)|t-s|^{\gamma}\right\}\right)=1 .
$$

Lemma 6.1 (Gradinaru-Nourdin [11]) Let $g: \mathbb{R} \rightarrow \mathbb{R}$ be a function satisfying

$$
|g(x)-g(y)| \leq C|x-y|^{a}\left(1+x^{2}+y^{2}\right)^{b}, \quad(C>0,0<a \leq 1, b>0),
$$

for all $x, y \in \mathbb{R}$ and let $X$ be a locally Hölder continuous paths process with index $\gamma \in(0,1)$. Assume that $V$ is a bounded variation continuous paths process. Set

$$
X_{\varepsilon}^{g}(t)=\int_{0}^{t} g\left(\frac{X_{s+\varepsilon}-X_{s}}{\varepsilon^{\gamma}}\right) d s
$$

for $t \geq 0$, $\varepsilon>0$. If for each $t \geq 0$, as $\varepsilon \rightarrow 0$,

$$
\left\|X_{\varepsilon}^{g}(t)-V_{t}\right\|_{L^{2}}^{2}=O\left(\varepsilon^{\alpha}\right)
$$


with $\alpha>0$, then $\lim _{\varepsilon \rightarrow 0} X_{\varepsilon}^{g}(t)=V_{t}$ almost surely, for any $t \geq 0$, and if $g$ is non-negative, for any continuous stochastic process $\left\{Y_{t}: t \geq 0\right\}$,

$$
\lim _{\varepsilon \rightarrow 0} \int_{0}^{t} Y_{s} g\left(\frac{X_{s+\varepsilon}-X_{s}}{\varepsilon^{\gamma}}\right) d s \rightarrow \int_{0}^{t} Y_{s} d V_{s}
$$

almost surely, uniformly in t on each compact interval.

Proposition 6.1 Let $f \in C^{1}(\mathbb{R})$. We have

$$
[f(B), B]_{t}^{(T Q)}=\int_{0}^{t} f^{\prime}\left(B_{s}\right) \frac{d s}{\sqrt{2 \pi s}}
$$

and, in particular, we have

$$
[B, B]_{t}^{(T Q)}=\sqrt{\frac{2 t}{\pi}}
$$

for all $t \geq 0$.

Proof By Lemma 6.1 and the Hölder continuity of the solution $u(t, x)$, it is enough to show that the estimate

$$
\left\|B_{t}^{\varepsilon}-\sqrt{\frac{2 t}{\pi}}\right\|_{L^{2}}^{2}=O\left(\varepsilon^{\alpha}\right)
$$

holds, for each $t \geq 0$, with some $\alpha>0$, as $\varepsilon \rightarrow 0$, where

$$
B_{t}^{\varepsilon}=\frac{1}{\sqrt{\varepsilon}} \int_{0}^{t}\left(B_{s+\varepsilon}-B_{s}\right)^{2} d \sqrt{s} .
$$

We have

$$
E\left|B_{t}^{\varepsilon}-\sqrt{\frac{2 t}{\pi}}\right|^{2}=\frac{1}{\varepsilon} \int_{0}^{t} \int_{0}^{t} A_{\varepsilon}(s, r) d \sqrt{s} d \sqrt{r}
$$

for $t \geq 0$ and $\varepsilon>0$, where

$$
\begin{aligned}
A_{\varepsilon}(s, r):= & E\left(\left(B_{s+\varepsilon}-B_{s}\right)^{2}-\sqrt{\frac{2 \varepsilon}{\pi}}\right)\left(\left(B_{r+\varepsilon}-B_{r}\right)^{2}-\sqrt{\frac{2 \varepsilon}{\pi}}\right) \\
= & E\left(B_{s+\varepsilon}-B_{s}\right)^{2}\left(B_{r+\varepsilon}-B_{r}\right)^{2}+\frac{2 \varepsilon}{\pi} \\
& -\sqrt{\frac{2 \varepsilon}{\pi}} E\left(\left(B_{s+\varepsilon}-B_{s}\right)^{2}+\left(B_{r+\varepsilon}-B_{r}\right)^{2}\right) .
\end{aligned}
$$

Define the function $\phi_{s}: \mathbb{R}_{+} \rightarrow \mathbb{R}_{+}$by

$$
\phi_{s}(x)=\frac{1}{\sqrt{2 \pi}}(\sqrt{2(s+x)}-2 \sqrt{2 s+x}+\sqrt{2 s})
$$


for every $s>0$. Then we have

$$
E\left[\left(B_{s+\varepsilon}-B_{s}\right)^{2}\right]=\frac{1}{\sqrt{2 \pi}}(\sqrt{2(s+\varepsilon)}-2 \sqrt{2 s+\varepsilon}+2 \sqrt{\varepsilon}+\sqrt{2 s})=\phi_{s}(\varepsilon)+\sqrt{\frac{2 \varepsilon}{\pi}}
$$

for all $s>0$. Noting that

$$
\begin{aligned}
& E\left[\left(B_{s+\varepsilon}-B_{s}\right)^{2}\left(B_{r+\varepsilon}-B_{r}\right)^{2}\right] \\
& \quad=E\left[\left(B_{s+\varepsilon}-B_{s}\right)^{2}\right] E\left[\left(B_{r+\varepsilon}-B_{r}\right)^{2}\right]+2\left(E\left[\left(B_{s+\varepsilon}-B_{s}\right)\left(B_{r+\varepsilon}-B_{r}\right)\right]\right)^{2}
\end{aligned}
$$

for all $r, s \geq 0$ and $\varepsilon>0$, we get

$$
A_{\varepsilon}(s, r)=\phi_{s}(\varepsilon) \phi_{r}(\varepsilon)+2\left(\mu_{s, r}\right)^{2},
$$

where $\mu_{s, r}:=E\left[\left(B_{s+\varepsilon}-B_{s}\right)\left(B_{r+\varepsilon}-B_{r}\right)\right]$. Now, let us estimate the function

$$
\phi_{s}(\varepsilon)=\frac{1}{\sqrt{2 \pi}}(\sqrt{2(s+\varepsilon)}-2 \sqrt{2 s+\varepsilon}+\sqrt{2 s}) .
$$

Clearly, one can see that

$$
\lim _{x \rightarrow 0} \frac{1-2 \sqrt{1-x / 2}+\sqrt{1-x}}{x^{2}}=-\frac{1}{16}
$$

and the continuity of the function $x \mapsto 1-2 \sqrt{1-x / 2}+\sqrt{1-x}$ implies that

$$
\left|\phi_{s}(\varepsilon)\right|=\sqrt{2(s+\varepsilon)}|1-2 \sqrt{1-x / 2}+\sqrt{1-x}| \leq C \frac{\varepsilon^{2}}{(s+\varepsilon)^{3 / 2}} \leq C \frac{\varepsilon^{\frac{1}{2}+\beta}}{(s+\varepsilon)^{\beta}}
$$

with $x=\frac{\varepsilon}{s+\varepsilon}$ and $0<\beta<\frac{1}{2}$, which gives

$$
\frac{1}{\varepsilon} \int_{0}^{t} \int_{0}^{t}\left|\phi_{s}(\varepsilon) \phi_{r}(\varepsilon)\right| d \sqrt{s} d \sqrt{r} \leq C t^{1-2 \beta} \varepsilon^{2 \beta} .
$$

It follows from Lemma 2.4 that there is a constant $\alpha>0$ such that

$$
\lim _{\varepsilon \downarrow 0} \frac{1}{\varepsilon^{1+\alpha}} \int_{0}^{t} \int_{0}^{t} A_{\varepsilon}(s, r) d \sqrt{s} d \sqrt{r}=0
$$

for all $t>0$, which gives the desired estimate

$$
\left\|B_{t}^{\varepsilon}-\sqrt{\frac{2 t}{\pi}}\right\|_{L^{2}}^{2}=O\left(\varepsilon^{\alpha}\right) \quad(\varepsilon \rightarrow 0)
$$

for each $t \geq 0$ and some $\alpha>0$. This completes the proof.

\section{Consider the decomposition}

$$
I_{\varepsilon}^{2}(f, x, t)=\frac{1}{\sqrt{\varepsilon}} \int_{0}^{t} f\left(B_{s+\varepsilon}\right)\left(B_{s+\varepsilon}-B_{s}\right) \frac{d s}{2 \sqrt{s}}-\frac{1}{\sqrt{\varepsilon}} \int_{0}^{t} f\left(B_{s}\right)\left(B_{s+\varepsilon}-B_{s}\right) \frac{d s}{2 \sqrt{s}}
$$




$$
\equiv I_{\varepsilon}^{2,+}(f, x, t)-I_{\varepsilon}^{2,-}(f, x, t)
$$

for $\varepsilon>0$, and by estimating the two terms in the right hand side above in $L^{2}(\Omega)$ one can structure the next Banach space:

$$
\mathscr{H}_{*}=\left\{f: \text { Borel functions on } \mathbb{R} \text { such that }\|f\|_{\mathscr{H}_{*}}<\infty\right\},
$$

where

$$
\|f\|_{\mathscr{H}_{*}}^{2}:=\frac{1}{\sqrt[4]{4 \pi}} \int_{0}^{T} \int_{\mathbb{R}}|f(z)|^{2} e^{-\frac{z^{2} \sqrt{\pi}}{2 \sqrt{s}}} \frac{d z d s}{s^{3 / 4}} \equiv \int_{0}^{T} E\left|f\left(B_{s}\right)\right|^{2} \frac{d s}{2 \sqrt{s}} .
$$

Clearly, $\mathscr{H}_{*}=L^{2}(\mathbb{R}, \mu(d z))$ with

$$
\mu(d z)=\left(\frac{1}{\sqrt[4]{4 \pi}} \int_{0}^{T} e^{-\frac{z^{2} \sqrt{\pi}}{2 \sqrt{s}}} \frac{d s}{s^{3 / 4}}\right) d z,
$$

and $\mathscr{H}_{*}$ includes all functions $f$ satisfying the condition

$$
|f(x)| \leq C e^{\beta x^{2}}, \quad x \in \mathbb{R}
$$

with $0 \leq \beta<\frac{\sqrt{\pi}}{4 \sqrt{T}}$. In the same way as proving Theorem 3.1 and by smooth approximation one can introduce the following result.

Theorem 6.1 The PQC $[f(B), B]^{(T Q)}$ exists and

$$
E\left|[f(B), B]_{t}^{(T Q)}\right|^{2} \leq C\|f\|_{\mathscr{H}_{*}}^{2}
$$

for all $f \in \mathscr{H}_{*}$ and $t \in[0, T]$. Moreover, if $F$ is an absolutely continuous function such that

$$
|F(x)|,\left|F^{\prime}(x)\right| \leq C e^{\beta x^{2}}, \quad x \in \mathbb{R}
$$

with $0 \leq \beta<\frac{\sqrt{\pi}}{4 \sqrt{T}}$, then the following Itô type formula holds:

$$
F\left(B_{t}\right)=F(0)+\int_{0}^{t} F^{\prime}\left(B_{s}\right) \delta B_{s}+\frac{1}{2 \sqrt{2}}\left[F^{\prime}(B), B\right]_{t}^{(T Q)}
$$

for all $t \in[0, T]$.

Recall that Russo and Tudor [27] have showed that $B=\left\{B_{t}=u(t, \cdot), t \geq 0\right\}$ admits a local time $L(t, a) \in L^{2}(\lambda \times P)$ such that $(a, t) \mapsto L(a, t)$ is jointly continuous, where $\lambda$ denotes the Lebesgue measure, since $B=\left\{B_{t}=u(t, \cdot), t \geq 0\right\}$ is a bi-fractional Brownian motion for every $x \in \mathbb{R}$. Define the weighted local time $\mathscr{L}$ of $B=\left\{B_{t}=u(t, \cdot), t \geq 0\right\}$ by

$$
\mathscr{L}(x, t)=\int_{0}^{t} \frac{1}{2 \sqrt{\pi s}} d_{s} L(s, x) \equiv \int_{0}^{t} \delta\left(B_{s}-x\right) \frac{d s}{2 \sqrt{\pi s}}
$$


for $t \geq 0$ and $x \in \mathbb{R}$, where $\delta$ is the Dirac delta function. Then the occupation formula

$$
\int_{0}^{t} \psi\left(B_{s}, s\right) \frac{d s}{2 \sqrt{\pi s}}=\int_{\mathbb{R}} d a \int_{0}^{t} \psi(a, s) \mathscr{L}(a, d s)
$$

holds for every continuous and bounded function $\psi: \mathbb{R} \times \mathbb{R}_{+} \rightarrow \mathbb{R}$ and any $x \geq 0$. As in Sect. 5, we can show that the integral

$$
\int_{\mathbb{R}} f_{\Delta}(x) \mathscr{L}(d x, t):=\sum_{j} f_{j}\left[\mathscr{L}\left(a_{j}, t\right)-\mathscr{L}\left(a_{j-1}, t\right)\right]
$$

is well-defined and

$$
\int_{\mathbb{R}} f_{\Delta}(x) \mathscr{L}(d x, t)=-\frac{1}{\sqrt{2}}\left[f_{\triangle}(B), B\right]_{t}^{(T Q)}
$$

almost surely, for all $f_{\triangle}=\sum_{j} f_{j} 1_{\left(a_{j-1}, a_{j}\right]} \in \mathscr{E}$. By the denseness of $\mathscr{E}$ in $\mathscr{H}_{*}$ one can define

$$
\int_{\mathbb{R}} f(x) \mathscr{L}(d x, t):=\lim _{n \rightarrow \infty} \int_{\mathbb{R}} f_{\Delta, n}(x) \mathscr{L}(d x, t)
$$

for any $f \in \mathscr{H}_{*}$, where $\left\{f_{\triangle, n}\right\} \subset \mathscr{E}$ and

$$
\lim _{n \rightarrow \infty} f_{\triangle, n}=f
$$

in $\mathscr{H}$. Moreover, the Bouleau-Yor type formula

$$
[f(B), B]_{t}^{(T Q)}=-\sqrt{2} \int_{\mathbb{R}} f(x) \mathscr{L}(d x, t)
$$

holds, almost surely, for all $f \in \mathscr{H}_{*}$.

Corollary 6.1 (Tanaka formula) For any $x \in \mathbb{R}$ we have

$$
\left|B_{t}-x\right|=|x|+\int_{0}^{t} \operatorname{sign}\left(B_{s}-x\right) \delta B_{s}+\mathscr{L}(x, t)
$$

\section{Acknowledgements}

The authors are thankful to anonymous referees for their careful corrections, helpful suggestions, and valuable comments on the original version of this paper.

\section{Funding}

The project was sponsored by NSFC (Nos. 11971101, 11571071, 11626033, 11701589), Innovation Program of the Shanghai Municipal Education Commission (12ZZ063), Natural Science Foundation of Anhui Province (1808085MA02) and Quality engineering of Anhui Education (2018jyssf045)

\section{Availability of data and materials}

Data sharing not applicable to this article as no datasets were generated or analyzed during the current study.

\section{Competing interests}

The authors declare that there is no conflict of interests regarding the publication of this paper. 


\section{Author details}

${ }^{1}$ Department of Mathematics, College of Science, Bengbu University, Bengbu, P.R. China. ${ }^{2}$ Department of Statistics, College of Science, Donghua University, Shanghai, P.R. China. ${ }^{3}$ School of Statistics and Mathematics, Zhejiang Gongshang University, Hangzhou, China.

\section{Publisher's Note}

Springer Nature remains neutral with regard to jurisdictional claims in published maps and institutional affiliations.

\section{Received: 1 January 2020 Accepted: 18 May 2020 Published online: 29 May 2020}

\section{References}

1. Alós, E., Mazet, O., Nualart, D.: Stochastic calculus with respect to Gaussian processes. Ann. Probab. 29, 766-801 (2001)

2. Balan, R., Kim, D.: The stochastic heat equation driven by a Gaussian noise: germ Markov property. Commun. Stoch. Anal. 2, 229-249 (2008)

3. Bouleau, N., Yor, M.: Sur la variation quadratique des temps locaux de certaines semimartingales. C. R. Acad. Sci., Sér. 1 Math. 292, 491-494 (1981)

4. Da Prato, G., Jentzen, A., Röckner, M.: A mild Itô formula for SPDEs (2012). arXiv:1009.3526

5. Deya, A., Tindel, S.: Malliavin Calculus for Fractional Heat Equation, Malliavin Calculus and Stochastic Analysis, 361-384. Springer Proc. Math. Stat., vol. 34. Springer, New York (2013)

6. Eisenbaum, N.: Integration with respect to local time. Potential Anal. 13, 303-328 (2000)

7. Errami, M., Russo, F.: n-covariation, generalized Dirichlet processes and calculus with respect to finite cubic variation process. Stoch. Process. Appl. 104, 259-299 (2003)

8. Feng, C.R., Zhao, H.Z.: Two-parameters p, q-variation paths and integrations of local times. Potential Anal. 25, 165-204 (2006)

9. Föllmer, H., Protter, P., Shiryayev, A.N.: Quadratic covariation and an extension of Itô's formula. Bernoulli 1, 149-169 (1995)

10. Geman, D., Horowitz, J.: Occupation densities. Ann. Probab. 8, 1-67 (1980)

11. Gradinaru, M., Nourdin, I.: Approximation at first and second order of m-order integrals of the fractional Brownian motion and of certain semimartingales. Electron. J. Probab. 8, 1-26 (2003)

12. Gradinaru, M., Nourdin, I., Tindel, S.: Ito's- and Tanaka's-type formulae for the stochastic heat equation: the linear case. J. Funct. Anal. 228, 114-143 (2005)

13. Houdré, C., Villa, J.: An example of infinite dimensional quasi-helix. In: Stochastic Models, Mexico City, 2002. Contemp. Math., vol. 336, pp. 195-201. Amer. Math. Soc., Providence (2003)

14. Lanconelli, A.: White noise approach to the Itô formula for the stochastic heat equation. Commun. Stoch. Anal. 1 311-320 (2007)

15. Lanconelli, A.: A remark on the renormalized square of the solution of the stochastic heat equation and on its associated evolution. Infin. Dimens. Anal. Quantum Probab. Relat. Top. 12, 497-502 (2009)

16. Lei, P., Nualart, D.: A decomposition of the bi-fractional Brownian motion and some applications. Stat. Probab. Lett. 79, 619-624 (2009)

17. León, J.A., Tindel, S.: Itô formula for linear fractional PDEs. Stochastics 80, 427-450 (2008)

18. Moret, S., Nualart, D.: Quadratic covariation and Itô's formula for smooth nondegenerate martingales. J. Theor. Probab. $13,193-224(2000)$

19. Mueller, C., Tribe, R.: Hitting probabilities of a random string. Electron. J. Probab. 7, 10 (2002)

20. Nualart, D.: Malliavin Calculus and Related Topics, 2nd edn. Springer, New York (2006)

21. Nualart, D., Vuillermot, P.-A.: Variational solutions for partial differential equations driven by a fractional noise. J. Funct. Anal. 232, 390-454 (2006)

22. Ouahhabi, H., Tudor, C.A.: Additive functionals of the solution to fractional stochastic heat equation. J. Fourier Anal. Appl. 19, 777-791 (2013)

23. Pardoux, E.: Two-sided stochastic calculus for SPDEs. In: Stochastic Partial Differential Equations and Applications, Trento, 1985. Lecture Notes in Math., vol. 1236, pp. 200-207. Springer, Berlin (1987)

24. Peskir, G.: A change-of-variable formula with local time on curves. J. Theor. Probab. 18, 499-535 (2005)

25. Pospisil, J., Tribe, R.: Parameter estimation and exact variations for stochastic heat equations driven by space-time white noise. Stoch. Anal. Appl. 4, 830-856 (2007)

26. Rogers, C.G., Walsh, J.B.: Local time and stochastic area integrals. Ann. Probab. 19, 457-482 (1991)

27. Russo, F., Tudor, C.A.: On bifractional Brownian motion. Stoch. Process. Appl. 116, 830-856 (2006)

28. Russo, F., Vallois, P.: Itô formula for $\mathcal{C}^{1}$-functions of semimartingales. Probab. Theory Relat. Fields 104, 27-41 (1996)

29. Russo, F., Vallois, P.: Stochastic calculus with respect to a continuous finite quadratic variation process. Stoch. Stoch. Rep. 70, 1-40 (2000)

30. Swanson, J.: Variations of the solution to a stochastic heat equation. Ann. Probab. 35, 2122-2159 (2007)

31. Torres, S., Tudor, C.A., Viens, F.G.: Quadratic variations for the fractional-colored stochastic heat equation. Electron. J. Probab. 19(76), 1-51 (2014)

32. Tudor, C.A.: Chaos expansion and regularity of the local time of the solution to the stochastic heat equation with additive fractional-colored noise. Taiwan. J. Math. 17, 1765-1777 (2013)

33. Tudor, C.A., Xiao, Y.: Some path properties of bi-fractional Brownian motion. Bernoulli 13, 1023-1052 (2007)

34. Tudor, C.A., Xiao, Y.: Sample paths of the solution to the fractional-colored stochastic heat equation. Stoch. Dyn. 17(03), 1750004 (2017)

35. Yan, L., Gao, B., Liu, J.: The Bouleau-Yor identity for a bi-fBm. Stochastics 86, 382-414 (2014)

36. Yan, L., Liu, J., Chen, C.: The generalized quadratic covariation for fractional Brownian motion with Hurst index less than 1/2. Infin. Dimens. Anal. Quantum Probab. Relat. Top. 17(4), 32 (2014) 
37. Yan, L., Liu, J., Yang, X.: Integration with respect to fractional local time with Hurst index $1 / 2<H<1$. Potential Anal. 30 , 115-138 (2009)

38. Zambotti, L.: Itô-Tanaka's formula for stochastic partial differential equations driven by additive space-time white noise. In: SPDEs and Applications VII. Lect. Notes Pure Appl. Math., vol. 245, pp. 337-347. Chapman \& Hall, Boca Raton (2006)

Submit your manuscript to a SpringerOpen ${ }^{\circ}$ journal and benefit from:

- Convenient online submission

- Rigorous peer review

- Open access: articles freely available online

- High visibility within the field

- Retaining the copyright to your article

Submit your next manuscript at $\gg$ springeropen.com 